II. Genealogie und Evolution 


\section{HUBERT THÜRING}

"Kraft, oder so was"

Poetik und Wissen(schaft) des Lebens in der Rahmenerzählung von Gottfried Kellers Sinngedicht

\section{Von Gott zur Natur zum Leben}

Die Natur und ihre Wissenschaften bilden für Gottfried Keller seit seinen literarischen Anfängen eine zentrale Referenz seiner Poetik, verstanden im weiten Sinn auch als Philosophie der Dichtung und gedichtete Philosophie. In der Forschung ist Kellers Bezug zur Natur zunächst vor allem als Ablösung für die vom Feuerbach'schen Materialismus verdrängte Gottesreferenz betrachtet worden. ${ }^{1}$ Demnach ist Religion selbst Produkt einer anthropologischen Naturkraft, mittels welcher der Mensch sich Umgang mit und Zugang zu der nicht unmittelbar erfahrbaren Natur verschafft. Dies gilt konsequenterweise auch für jene Natur, die seit der zweiten Hälfte des 18. Jahrhunderts von Philosophie und Wissenschaft als quasireligiöse Referenz zu enormer Wirkungsmacht gebracht wird. ${ }^{2}$ Die Einsicht, dass die Erfahrung der Natur vermittelt bleibt, die neuen Erkenntnisse über sie bloß relativen Geltungswert haben und eine Verortung des Menschen als Teil der Natur problematisch ist, entwickelt Keller erst allmählich, indem er den beobachteten Wandel in den Naturwissenschaften thematisiert und reflektiert. Dabei verschiebt sich die Leitreferenz seiner Poetik von der Natur zum Leben, dessen Begriff gegenüber den wissenschaftlichen Zugriffen eine Resistenz behauptet.

Die zeitgenössischen Naturwissenschaften, die den Wandel der Welterklärungen mit den entsprechenden Konsequenzen für das Erzählen während der ganzen Schaffenszeit Kellers maßgeblich bestimmen, werden in seinem Werk neben dem Grünen Heinrich (1854-1855, 1879-1880/1889) nur noch im Sinngedicht (1879-1881) so direkt, konkret und gewichtig thematisiert, und erst in letzterem treten sie, wenigstens in der Rahmengeschichte, mit dem Protagonisten in den Vordergrund. Während die Quellen- und Dis-

1 Vgl. Karl Fehr: Gottfried Keller. Aufschlüsse und Deutungen, München 1972, S. 8-28 u. 86-91.

2 Vgl. Gerhard Kaiser: Gottfried Keller. Das gedichtete Leben, Berlin 1995, vgl. S. 158-162 u. 172-174. 
kursbeziehungen und die poetischen Konsequenzen für den Grünen Heinrich schon mehrfach breit und gründlich aufgearbeitet worden sind, hat sich die Forschung zum Sinngedicht vor allem auf die ausgestellten Bezüge zur Lichtphysik in der Rahmenerzählung und auf die teils offenkundigen, teils untergründigen motivischen und narrativen Beziehungen zum Darwinismus konzentriert. Der Lebensbegriff aber, der vor allem im Sinngedicht primäre Relevanz gewinnt, ist im Schatten des dominierenden Naturbegriffs geblieben und in seiner Bedeutung nicht erkannt worden. Die vorliegende Studie wird daher den Lebensbegriff im Sinngedicht ins Licht rücken.

Zunächst soll diese Verschiebung von der Natur- zur Lebensreferenz im Spiegel der Forschung zu Der Grüne Heinrich und zu Das Sinngedicht betrachtet werden, wo Natur- und Lebenswissen bzw. -wissenschaft am deutlichsten und dichtesten thematisiert werden (1). Auf der Basis der dabei gewonnenen Thesen werde ich daraufhin die Rahmenerzählung des Sinngedichts, die im engeren Sinn nur das erste Kapitel umfasst, textnah beobachten, indem ich die topische 'Bestückung, narrative Entwicklung und diskursive Vernetzung analysiere und kommentiere und dabei den Akzent des Lebenswissens herausarbeite (2). Einen ersten Teil der im Verlauf aufgeworfenen Fragen zum natur- und lebenswissenschaftlichen Wissen soll eine verdichtete Vorgeschichte einholen, die den besonderen Status des Lebensbegriffs aufzeigt und zum zeitgenössischen Materialismus hinführt (3). Dieser wird dann, wieder in näherem Zusammenhang mit Keller und dem Sinngedicht, anhand von Ludwig Feuerbach und Jacob Moleschott und mit Blick auf die jeweiligen miteinander zu vergleichenden Differenzierungen des Natur- und Lebensbegriffs dargestellt (4). In unmittelbarer Textnähe werde ich darauf Moleschotts Zürcher Antrittsvorlesung Licht und Leben (1856) als direkte Quelle und narrativ-argumentative Folie des Sinngedichts analysieren und den erkenntnispoetischen Status des Lebenswissens umreißen (5). Anhand der dabei problematisierten Momente von Zeit und Zufall wird ein letzter Abschnitt den Umgang des Textes mit dem Darwinismus skizzieren (6).

\section{I.1. Forschung zum Grünen Heinrich}

Gerhard Kaiser, Dominik Müller, Wolfgang Rohe und Philip Ajouri haben vor allem für den Grünen Heinrich gezeigt, wie die Natur zunächst, in der ersten Fassung (1854/55), als objektive Gegenreferenz zur subjektiven (religiösen) Schicksalsgläubigkeit die Ordnung und den Gang der Erzählwelt garantiert. Die Kenntnis des Materialismus von Ludwig Feuerbach und Jacob Moleschott und weiterer naturwissenschaftlicher Forschungen 
hat den religiös oder idealistisch genährten Glauben an einen teleologischen Weltprozess mit integriertem Einzelschicksal gebrochen. Untragbar ist dadurch auch eine Romanpoetik geworden, die auf eine Parallelisierung von Real- und Erzählteleologie und eine Engführung von Welt- und Individualbestimmung baut, wie sie theoretisch der Versuch über den Roman (1774) von Christian Friedrich von Blanckenburg und praktisch Wilhelm Meisters Lehrjahre (1795/96) von Goethe modelliert haben. Die Natur und ihre Gesetzlichkeit bleiben trotz der kontingenten Brechung der biographischen Linien, die Heinrichs persönlichen Schicksalsglauben als (anthropologisch notwendige) Projektion markiert, für die Narration als Referenz einer (kausalen) Ordnung und als Quelle von Bildern erhalten, und Heinrich klammert sich auch bei den kleinsten Wendungen des Lebens wider besseres Wissen umso fester an den göttlichen »Wunderglauben «. 3

In der zweiten Fassung (1879-1880/1889), die nun auch unter dem Einfluss der Evolutionstheorie von Charles Darwin steht, werden die Naturgesetzlichkeit, im Sinn einer unverrückbaren Ordnung, sowie die narrative Kausalität und Finalität durch das "offene Ende, die Rücknahme der Lebensreise als natürliche Form der Biographie, die Einfügung von Parallelgeschichten und Allegorien, die Rücknahme der Typologie und den Abbau von Zielvorstellungen" weiter aufgelöst; die "Anthropomorphismen", die "Konnotationen der Natur als etwas Göttlichem" verschwinden zugunsten der "Grausamkeit und Gleichgültigkeit der Natur«. ${ }^{4}$ Eine vertiefte Reflexion über den unberechenbaren "Lauf der Welt" geht einher mit der Markierung der Zufälle als Erzählereingriffe, die für die relative Finalität der Fiktion notwendig sind. ${ }^{5}$ Der innere Zusammenhang und eine gewisse kompositorische Geschlossenheit entstehen durch ein verdichtetes inneres Verweisnetz der narrativen und rhetorischen Elemente und Motive. ${ }^{6}$

Im Grünen Heinrich stellen, wie Rohe in seiner Analyse der ersten Fassung zeigt, "[s]owohl die Konversationsrunde bei Frau Margret als auch das Handwerkerkünstlertum Vater Lees« zunächst noch "anachronistische Entwürfe einer Integration der Naturwissenschaft in die Gesellschaft dar«?

Ebd., S. 172.

4 Philip Ajouri: Erzählen nach Darwin. Die Krise der Teleologie im literarischen Realismus: Friedrich Theodor Vischer und Gottfried Keller, Berlin/New York 2007, S. 335.

5 Vgl. ebd., S. 331.

6 Vgl. ebd., S. 276.

7 Wolfgang Rohe: Roman aus Diskursen. Gottfried Keller »Der grüne Heinrich" (Erste Fassung; 1854/55), München 1993, S. 151. 
Doch dann führt Heinrichs Besuch der »Vorträge über Anthropologie«, 8 für welche die Anthropologischen Vorträge von Jakob Henle ${ }^{9}$ (gehalten 1849) sowie Moleschotts Lehre der Nahrungsmittel (1850) und Kreislauf des Lebens (1852) die primären Referenzen bilden, zu einer differenzierten Auseinandersetzung mit der zeitgenössischen Wissenschaft. Heinrichs Vorhaben, seine neue 'Kunst der Gestalt mit physiologischem Wissen zu fundieren, lässt sich nicht in die Praxis umsetzen. In philosophischer Hinsicht integriert Heinrich einerseits das neue Wissen als Bestätigung der Perfektion von Gottes Schöpfung, andererseits stößt er sich an den Angriffen auf die Lehre vom freien Willen ${ }^{10}$ und münzt den materialistischen Determinismus in Vorsätze zur guten Lebensführung um. Während der Protagonist auf diese Weise an der Selbstbestimmung des Subjekts festhält, werden die neuen wissenschaftlichen und philosophischen Erkenntnisse neben anderen Diskursen und Praktiken von der Handlung und von der beobachtenden und kommentierenden Erzählstimme in ihrer Heterogenität referiert und performiert. So können Vegetationsvergleiche und Ernährungsverhalten, welche die Diskrepanz zwischen bewusster Absicht und triebhaftem Handeln zum Ausdruck bringen, mehr oder weniger direkt mit den genannten materialistischen Theorien der Ernährung und des Stoffwechsels in Zusammenhang gebracht werden. ${ }^{11}$ Starke Wertungen durch den Erzähler oder durch Figuren finden sich allenfalls in Karikaturen oder der Ablehnung von Extremen der Wissenschaftsgläubigkeit, auch Normalisierungen im Sinn einer Reduktion auf rein Technisches oder auf Religion, Theologie oder Philosophie werden mittels selbstentlarvender Vorführung unterlaufen.

Der Grüne Heinrich lässt also einerseits seinen Protagonisten beim Versuch, naturwissenschaftliches Wissen künstlerisch umzusetzen, scheitern, bringt dieses aber andererseits thematisch, motivisch und narrativ zur Darstellung, ohne ein neues "sinnorientierendes Ganzes" im Zeichen der Kunst zu entwerfen. ${ }^{12}$ Adornos These der Ästhetischen Theorie aufnehmend, wonach Wissenschaft und Kunst fundamental verschieden seien, die moderne Kunst jedoch, wenn sie gesellschaftliche Wahrheit beanspruche, sich jedem Gehalt öffnen müsse, resümiert Rohe, dass die »Gestaltungsleistung des `Grünen

8 Gottfried Keller: Der Grüne Heinrich [zweite Fassung] (1879-1880/1889), in: Ders.: Sämtliche Werke, Historisch-Kritische Ausgabe (HKKA), hg. von Walter Morgenthaler, Basel/Frankfurt a.M./Zürich 1997ff., Bd. 1-3 (2006), hg. von Karl Grob u.a., Bd. 3, S. 9ff.

9 Vgl. Jakob Henle: Anthropologische Vorträge, Erstes Heft, Braunschweig 1876.

10 Vgl. Keller: Der Grüne Heinrich [zweite Fassung], HKKA 3 (wie Anm. 8), S. $19 \mathrm{ff}$.

11 Vgl. Rohe: Roman aus Diskursen (wie Anm. 7), S. 162-167.

12 Ebd., S. 160. 
Heinrich « darin bestehe, "sich weder ins 'sakrosankte` Refugium des 'ästhetischen Prinzips` zurückgezogen, noch sich ins Adaptieren wissenschaftlicher Standards gerettet zu haben«, sondern die Heterogenität diskursiv wie strukturell zu verarbeiten. ${ }^{13}$ Dass der Roman dabei nicht seine Form verliert, liegt daran, dass in der ersten Fassung die Handlung noch von der biographischen Teleologie getragen wird und die Natur trotz der diskurs-perspektivischen Heterogenität als zentrale Referenz fungiert. Ajouris vergleichende Analyse hat gezeigt, dass die verstärkte Tendenz zur Auflösung der Erzählteleologie in der zweiten Fassung mit einer Verdichtung der Verweisstruktur von Motiven und Binnennarrativen einhergeht. Die Natur erscheint im Zeichen Darwins nicht mehr als fixe Referenz aller Diskurse, sondern als eine der Zeit unterworfene, sich verändernde Instanz, die ebenso Leben vernichtend wie Leben hervorbringend agiert.

Mithin scheint sich in der zweiten Fassung eine neue Referenz herauszubilden, die sich von der Naturreferenz unterscheidet und sich ihr bis zu einem gewissen Grad entgegensetzt. Diese Referenz bzw. dieser Referenzbereich ist das Leben. Neben dem Naturbegriff ist der Lebensbegriff in Kellers Poetologie seit seiner 'Konversion` zum Materialismus und Atheismus 1849 mit diesem einschlägigen Akzent präsent:

Er [Feuerbach] hat nichts als die Natur und wieder die Natur, er ergreift sie mit allen seinen Fibern in ihrer ganzen Tiefe und läßt sich weder durch Gott noch Teufel aus ihr herausreißen. Für mich ist die Hauptfrage die: Wird die Welt, wird das Leben prosaischer und gemeiner nach Feuerbach? Bis jetzt muß ich des bestimmtesten antworten: Nein! im Gegenteil, es wird alles klarer, strenger, aber auch glühender und sinnlicher. ${ }^{14}$

Obwohl philosophisch-ästhetisch kontextualisiert, erscheint das 'Leben kaum verbegrifflicht oder wissenschaftlich konturiert, sondern meint allgemein und in Überschneidung mit dem Naturbegriff die Sphäre der biologisch-organischen Natur, der Vitalität oder dann das zeitlich begrenzte sinnliche und geistige Dasein des Menschen. Die philosophisch und ästhetisch dominante Referenz scheint die Natur auch noch dort zu bilden, wo sie eigentlich das Leben im engeren biologischen Sinn und die menschliche Existenz im Ganzen bezeichnet:

13 Ebd., S. 171.

14 Gottfried Keller: Brief an Wilhelm Baumgartner, Heidelberg, 28. Januar 1849, in: Ders.: Gesammelte Briefe, vier Bände, hg. von Carl Helbling, Bern 1950, Bd. 1, S. 275. 
Nur für die Kunst und Poesie ist von nun an kein Heil mehr ohne vollkommene geistige Freiheit und ganzes glühendes Erfassen der Natur ohne alle Neben und Hintergedanken, und ich bin fest überzeugt, daß kein Künstler mehr eine Zukunft hat, der nicht ganz und ausschließlich sterblicher Mensch sein will. Daher ist mir auch meine neuere Entwicklung und Feuerbach für meine dramatischen Pläne und Hoffnungen weit wichtiger geworden, als für alle übrigen Beziehungen, weil ich deutlich fühle, daß ich die Menschennatur nun tiefer zu durchdringen und zu erfassen befähigt bin. Jedes dramatische Gedicht wird um so reiner und konsequenter sein, als nun der letzte Deus ex machina verbannt ist und das abgebrauchte Tragische wird durch den wirklichen und vollendeten Tod einen neuen Lebenskeim gewinnen. ${ }^{15}$

Trotz der Dominanz des Naturbegriffs lässt sich indes bereits hier eine Differenz zwischen 'Natur` und 'Leben` erkennen, die jedoch vorerst weder begrifflich noch poetisch produktiv wird. Kellers immanenter Naturbegriff orientiert sich zum einen an der aristotelischen Poetik der Nachahmung, die zunächst im Zeichen der Goethe'schen und Kant'schen Organologie differenziert worden ist und nun auch von der Stoffwechseltheorie eine materialistische Erweiterung und Vertiefung erfahren hat. Diese Entwicklung hat den vitalistisch-idealistischen Lebensbegriff, der um 1800 noch Hochkonjunktur hatte und weit in die erste Hälfte des 19. Jahrhunderts hineinwirkte, verdrängt. $^{16}$

Zwischen diesen diskursiven Bedingungen, die für die erste Fassung des Grünen Heinrich maßgebend sind, und denjenigen zur Zeit der zweiten Fassung liegen wiederum dreißig Jahre, in welche die Differenzierung des Materialismus und die Darwin'sche Formulierung der Selektionstheorie als Erklärung für die Entwicklung der Arten fällt. Die Materialismusdebatte erweitert die Stoffwechseltheorie um die Frage nach den Entstehungs- und

15 Keller: Brief an Wilhelm Baumgartner, Berlin, 27. März 1851, Bd. 1 (wie Anm. 14), S. 291.

16 Einen ästhetischen Ansatz zum Lebensbegriff bei Keller verfolgt Rebecca Lötscher in ihrer Masterarbeit Das Schöne leben. Zum Lebensgefïhl als Grundlage der Ästhetik - mit Kant, Cassirer und Kellers "Grünem Heinrich" (Basel 2011): Sie erarbeitet die Kant'sche Ästhetik entlang der Subjekt/Objekt-Beziehung, der Unterscheidung von Angenehmem/Schönem, Zweck/ Zweckmäßigkeit, bestimmendem/reflektierendem Urteil, Naturschönem/Kunstschönem und des >organischen Zusammenhangs dieser Kategorien in zum Teil dichtem Dialog mit der Erzählung von der existenziellen und künstlerischen Entwicklung Heinrich Lees. Dabei geht es nicht um eine einseitige Anwendung und Überprüfung, denn sie zeigt auf, wie der Roman diese Entwicklung auf der Figuren- und der Erzählerebene reflektiert. Mit der Entwicklung der Figur gelangt die Arbeit zum freien Spiel der Erkenntniskräfte, das sich im Verhältnis von ästhetischer Idee und Vernunftidee fortsetzt, einer Sphäre, in welche die Figur nur bedingt hineinreicht, während der Erzähler sich hier auf der philosophischen Höhe der Kant'schen Ästhetik bewegt. 
Entwicklungsbedingungen von Leben und den Möglichkeitsbedingungen von dessen Erkenntnis. Mit Darwin verschiebt sich der Fokus zwar auf die kontingente Interaktion der Lebewesen, denen die 'Natur auch feindlich erscheinen kann. Während dies, wie bereits erwähnt, zur weiteren Entteleologisierung beiträgt, bleibt die ästhetische Referenz der Natur als dynamische Gesetzmäßigkeit erhalten. Es sei dahingestellt, wie stark Keller bei der 'Revision des Grünen Heinrich darum bemüht war, diese Natur zugunsten eines Referenzfeldes des Lebens auszutreiben; jedenfalls ließen sich die diskursiven Veränderungen kaum darstellend oder reflektierend einbauen, zumal die durchgehende Autodiegese hier einschränkend wirkte.

Diese Diskursgeschichte erzählend einzuholen und eine neue reflektierte Ästhetik oder Poetik zu realisieren, welche die Natur nun entschieden in die Perspektive des (menschlichen) Lebens stellt, unternimmt die Rahmengeschichte des Sinngedichts. Der letzte Novellenzyklus Kellers ist neben dem Grünen Heinrich das einzige Werk, in dem die Wissenschaft derart in den Vordergrund rückt, hier sogar im Protagonisten Reinhart personifiziert.

\section{I.2. Forschung zum Sinngedicht}

In der Forschung hat vor allem die prominente Thematisierung des Darwinismus die Aufmerksamkeit auf sich gezogen, meistens verbunden mit der These von einem Antagonismus zwischen den rzwei Kulturen Wissenschaft und Literatur, der vor allem in der Rahmengeschichte thematisch entfaltet, aber auch in den einzelnen Novellen verschoben oder übertragen durchgespielt wird. ${ }^{17}$ Mit Fokus auf die Rahmengeschichte hat maßgeblich Gerhard Kaiser dargelegt, wie die beiden Kulturen und ihre typischen Prak-

17 Vgl. Kaiser: Gottfried Keller (wie Anm. 2), S. 503-514 u. 555; Ders.: Experimentieren oder Erzählen? Zwei Kulturen in Gottfried Kellers Sinngedicht, in: Jahrbuch der Deutschen Schillergesellschaft 45 (2001), S. 278-301. Zuvor hat Wolfgang Preisendanz (Gottfried Kellers Sinngedicht, in: Zeitschrift für deutsche Philologie 82 [1963], S. 129151) den Auszug des Naturwissenschaftlers aus dem Labor ins Feld der Liebe als Absage an zeitgenössische Ansprüche interpretiert, die Literatur habe sich an der Naturwissenschaft zu orientieren. Dagegen haben Kaiser (Das gedichtete Leben [wie Anm. 2], S. 562-576) und Rohe (Roman aus Diskursen [wie Anm. 7], S. 142-145) argumentiert, dass die Referenzen auf die Naturwissenschaften eine Eigenständigkeit behaupten und eine implizite Eigendynamik entfalten. Julius Rothenberg (Geheimnisvoll schöne Welt. $\mathrm{Zu}$ Gottfried Kellers "Sinngedicht" als antidarwinistischer Streitschrift, in: Zeitschrift für deutsche Philologie 95 [1976], S. 255-290) hat die zahlreichen und virtuosen Ausformungen des Kampf-ums-Dasein-Musters ausführlich untersucht, allerdings unter der einschränkenden Prämisse einer durchweg antidarwinistischen Haltung Kellers. 
tiken, Experimentieren und Erzählen, in einem weiten und dichten Beziehungsgeflecht von weiteren Antagonismen - insbesondere demjenigen der Geschlechter, aber auch fremd und eigen, arm und reich - miteinander verknüpft werden, ohne jedoch miteinander zu verschmelzen. Ausgehend von einer kursorischen Kommentierung der naturwissenschaftlichen Referenzen der Eingangsszenerie in Reinharts Studierzimmer, die Kaiser vornehmlich in der 'kalten Newton'schen Physik ansiedelt, analysiert er dann die Implikationen des »Liebeskriegs", den der Naturforscher Reinhart und die Gärtnerin und Leserin Lucie erzählend ausfechten. Das von Lucie verkörperte »lebendige Leben« scheint über die Wissenschaft zu siegen, ${ }^{18}$ während der gesichts- und geschichtslose Reinhart anscheinend nur den neusten biologischen Lehrsatz vom Fressen und Gefressenwerden beizusteuern weiß. Die im Erzählen gewonnene Erkenntnis, die Reinhart seine Existenz in eine Epoche ante lucem und post lucem (bezogen auf den lateinischen Wortstamm von Lucies Namen) einteilen lässt, ist eine literarische Erkenntnis, die gerade das Vorher und Nachher zu verbinden und auf eine Zukunft auszurichten vermag. Diese Erkenntnis verhält sich strukturell analog, aber diskursiv antithetisch zur biologischen Erkenntnis der Selektion, die das Wissen in ein ungültiges Vorher und ein gültiges Nachher teilt. ${ }^{19}$ Die beiden Erfahrungswelten des biologischen Wissens und des existenziellen Lebens werden Kaiser zufolge nicht in einer "umfassenden Integrationsinstanz" aufgehoben, sondern im Modus der »Umschlägigkeit« zugleich verbunden und getrennt. Der Modus entspreche Feuerbachs Erkenntnis, dass das »Wirkliche [...] im Denken nicht in ganzen Zahlen, sondern nur in Brüchen darstellbar" sei. ${ }^{20}$ Im Vergleich mit dem zeitgenössischen Naturalismus würden Kellers Kenntnisse der aktuellen Naturwissenschaft »leicht altmodisch" erscheinen. Anstatt, wie in Goethes Wahlverwandtschaften (1809), die tödliche Konsequenz der buchstäblichen Übersetzung von Wissenschaft in die Lebenspraxis vorzuführen oder, wie Zolas Les Rougon-Macquart (1871-1893), die tödliche Konsequenz des Lebens selbst quasiexperimentell aufzuzeigen, werde das Experimentieren im und als Erzählen in einem "Zwischenreich" erprobt, gerade um die tödliche Konsequenz im Leben auszusetzen oder aufzuschieben. ${ }^{21}$ Das von Lucie und Reinhart gemeinsam bestandene »Rettungsaben-

18 Kaiser: Experimentieren oder Erzählen? (wie Anm. 17), S. 291.

19 Vgl. ebd., S. 297.

20 Ludwig Feuerbach: Grundsätze der Philosophie der Zukunft, in: Ders.: Gesammelte Werke, hg. von Werner Schuffenhauer, Berlin 1967ff., Bd. 9, S. 330.; vgl. Kaiser: Experimentieren oder Erzählen? (wie Anm. 17), S. 297.

21 Ebd., S. 298-301. 
teuer« der Schlange vor dem Krebs setzt sowohl den darwinistischen Kampf ums Dasein als auch das Erzählen zugunsten des gelebten Lebens (d.h. als biologisches Phänomen und kulturelle Praxis) voraus. ${ }^{22}$

\section{I.3. Zusammenfassung und Thesen}

Überblickt man die Forschung zum Motiv, Begriff und Diskurs der Natur und Naturwissenschaften in Kellers einschlägigen Werken, so scheint sie eine Verschiebung oder Verlagerung vom Natur zum Lebensbegriff nachzuzeichnen, die noch nicht explizit herausgearbeitet worden ist. Die Konturen des Lebensbegriffs hat bislang Kaiser am deutlichsten hervortreten lassen, dabei aber die historischen und zeitgenössischen diskursiven Referenzen zu wenig befragt und zum Teil mit literarischen und philosophischen Topoi verstellt. Dadurch ist ihm, wie auch den anderen Forschungen zum Sinngedicht, entgangen, dass die Rahmenerzählung eine Diskursgeschichte der Wissenschaften von der Natur zum Leben darstellt. Dies geschieht zu einem guten Teil mittels Konstellationen im Raum, d.h. mittels der Ausstattung und Bestückung des Laboratoriums, aber auch in den Reinhart zugedachten Überlegungen zum Zusammenhang der Dinge und schließlich durch Reinharts Handeln. Im Modus der Gleichzeitigkeit des Ungleichzeitigen und der Zeitraffung wird eine Geschichte der Wissenschaft von der Alchemie über die Newton'sche Physik, Goethes organologische Biologie, den idealistischen Vitalismus, den Materialismus Feuerbachs und Moleschotts bis zum Darwinismus thematisch oder performativ erzählt oder miterzählt. Es lässt sich zeigen, dass Kellers Lebenswissen nicht nur von anderen diskursiven Bereichen her abgeleitet und erschlossen werden kann, d.h. vom religiösen, physikalischen oder materialistischen Naturbegriff, vom gelebten Leben der Figuren oder von der poetisch-narrativen Komposition, sondern auch unmittelbarere Bezeichnungen und Darstellungen erfährt. Dabei bleibt der Materialismus mit seiner philosophischen Maxime der Einheit von Kraft und Stoff (Ludwig Büchner, 1855) und der Chemie des Stoffwechsels die maßgebliche Referenz, zu welcher der Darwinismus mit seiner strukturaltemporalen Dynamik gewissermaßen das Pendant liefert. In diesem Feld des modernen Wissens initiiert die Rahmengeschichte des Sinngedichts das Experiment, das mit seinen beiden Polen der Physik und der Dichtung den weiteren historischen und diskursiven Horizont markiert.

22 Gottfried Keller: Das Sinngedicht (1879-1881), HKKA, Bd. 7 (1998), hg. von Walter Morgenthaler u.a., S. 3-329, S. 324f. 


\section{Topische Bestückung, narrative Entwicklung und diskursive Vernetzung der Rahmenerzählung des Sinngedichts}

Bei der Analyse und Interpretation der Rahmenerzählung, die sich eigentlich über die ersten fünf kurzen Kapitel, die Zwischenstücke zwischen den Novellen und das letzte Kapitel erstreckt, werde ich mich auf das erste Kapitel sowie auf eine Szene des letzten Kapitels beschränken, wobei hier zunächst das erste Kapitel und im letzten Abschnitt (6) dann die Szene betrachtet wird.

»Vor etwa fünfundzwanzig Jahren«, so beginnt der Erzähler die Rahmenerzählung mit einer umständlichen zeitlichen Situierung,

als die Naturwissenschaften eben wieder auf einem höchsten Gipfel standen, obgleich das Gesetz der natürlichen Zuchtwahl noch nicht bekannt war, öffnete Herr Reinhart eines Tages seine Fensterläden und ließ den Morgenglanz, der hinter den Bergen hervorkam, in sein Arbeitsgemach, und mit dem Frühgolde wehte eine frische Sommermorgenluft daher und bewegte kräftig die schweren Vorhänge und die schattigen Haare des Mannes. ${ }^{23}$

Der Erzähler legt den (vorgreifenden) Anachronismus zwischen erzählter Zeit und Zeitpunkt des Erzählens bloß und verweist damit zugleich auch auf jene weniger auffälligen Anachronismen, die in einem erweiterten Sinn als Verbindungen, Überlagerungen, Vermischungen von Motiven, Narrativen und Modellen aus verschiedenen Zeiten bestehen. Sie sind als potenzielle Gleichzeitigkeit des Ungleichzeitigen auch dann wirksam, wenn sie in der Unterscheidung von sfrüher und heuter die Handlung situieren und die erzählte Zeit strukturieren. Das gehört natürlich zu den verbürgten Mitteln der Poetik, erzeugt hier aber durch die Dichte der Elemente einen Effekt der Künstlichkeit, die den Rahmentext selbst als experimentelles Arrangement markiert, das gegen Ende des ersten Kapitels in das Handlungsexperiment im Zeichen des Sinnspruchs von Logau überführt wird.

In der "Studierstube« verdichtet der Erzähler Epochen der Naturwissenschaft, indem er das zeitgenössische Labor des Physikers, Geologen, Chemikers und Biologen von der Alchemisten-Gruft des vormodernen "Doctor Faust[us]« abhebt, diese aber zugleich als Vorgeschichte miteinbezieht:

Statt der malerischen Esse, der ungeheuerlichen Kolben und Kessel, gab es da nur feine Spirituslampen und leichte Glasröhren, Porzellanschalen und Fläschchen mit geschliffenem Verschlusse, angefüllt mit Trockenem und Flüssigem aller Art, mit

23 Ebd., S. 9. 
Säuren, Salzen und Krystallen. Die Tische waren bedeckt mit geognostischen Karten, Mineralien und hölzernen Feldspathmodellen; Schichten gelehrter Jahrbücher in allen Sprachen belasteten Stühle und Divans, und auf den Spiegeltischchen glänzten physikalische Instrumente in blankem Messing. Kein ausgestopftes Monstrum hing an räucherigem Gewölbe, sondern bescheiden hockte ein lebendiger Frosch in einem Glase und harrte seines Stündleins, und selbst das übliche Menschengerippe in der dunkeln Ecke fehlte, wogegen eine Reihe von Menschen- und Tierschädeln so weiß und appetitlich aussah, daß sie eher den Nippsachen eines Stutzers glichen, als dem unheimlichen Hokuspokus eines alten Laboranten. Statt bestaubter Herbarien sah man einige feine Bogen mit Zeichnungen von Pflanzengeweben, statt schweinslederner Folianten englische Prachtwerke in gepreßter Leinwand. ${ }^{24}$

Im Modus der Kontrastierung tritt einerseits die Differenz zwischen vormoderner und aufgeklärter Wissenschaft in den Vordergrund. Andererseits entsteht mit der Gleichzeitigkeit des Ungleichzeitigen eine Atmosphäre, in der die Gegenwart selbst als historisch bedingt und kontingent erscheint, was auch die ironischen Einsprengsel des Erzählers bestätigen. In die verdichtete Zeit zu integrieren sind mithin auch die eingangs ironisch erwähnten »höchsten Gipfel« der Naturwissenschaft sowie, als expliziter Anachronismus, die "natürliche[] Zuchtwahl«. Auf welche Wissenschaften und besonderen Entdeckungen oder Erkenntnisse sich diese "Gipfel« beziehen, führt die darauf folgende Schilderung der Person und Tätigkeit Reinharts nicht mit weiteren oder genaueren Namen und Daten aus. Auch hier steht die narrative Erzeugung einer Atmosphäre von naturwissenschaftlicher Tätigkeit mittels Verdichtung von Versatzstücken und Strömungen verschiedenen Datums im Vordergrund. Dennoch und gerade deshalb laden die mit relativierenden Wendungen versehenen Indizien zur Rekonstruktion von Zusammenhängen und deren Deutung ein.

Dass der Held einer positiven und exakten Naturwissenschaft verpflichtet ist, unterstreicht der Befund, dass die Bücher und Hefte nur "Zahlensäulen und Logarithmen« enthalten und »[k]ein einziges Buch" von moralischen, sentimentalen oder ästhetischen Dingen handelt. Reinhart experimentiert mit einem »sinnreiche[n] Apparat, allwo ein Sonnenstrahl eingefangen und durch einen Krystallkörper geleitet wurde, um sein Verhalten in demselben zu zeigen und womöglich das innerste Geheimnis solcher durchsichtigen Bauwerke zu beleuchten«. Nachdem er auch an diesem Morgen die »schöne Welt mit allem, was draußen lebte und webte«, wieder ausgeschlossen und das Zimmer bis auf ein »kleines Löchlein" verdunkelt hat, schickt sich Reinhart an, den einfallenden "Strahl sorgfältig auf die Tortur zu spannen«

24 Ebd., S. 9f. 
und mit Blick durch eine »Röhre, den Rechenstift in der Hand«, »Zahlen auf Zahlen« zu schreiben wie "viele Tage« davor. ${ }^{25}$

Die Folie der Newton'schen Lichtphysik und die kritischen bis krass polemischen Auseinandersetzungen Goethes mit dieser "Folter des Lichts" in seiner Farbenlehre (1810) ist unverkennbar und als solche auch quellenmäßig identifiziert und ansatzweise kommentiert. ${ }^{26}$ Goethe zufolge verkennt der physikalische Zugang zum Licht, der es als zusammengesetztes Phänomen erweisen möchte, dessen wahres Wesen: Das Licht ist ein unteilbares und, wie die personifizierenden Tropen nahelegen, lebendiges Wesen mit dem Vermögen, die Farbenwelt erscheinen zu lassen. ${ }^{27}$ Reinharts Experiment stellt offenbar das Newton'sche experimentum crucis nach, doch die Intention scheint eine spezifischere als das bloße Aufzeigen der Spektralfarben zu sein. Der Erzähler nimmt (ironische) Distanz, beschreibt in groben Zügen den Apparat und das Verhalten des Experimentators und stellt die Vermutung an ("womöglich"), dass es um das Geheimnis des Kristallkörpers gehen könnte. - Bestimmteres gibt der Text direkt nicht her, vielmehr stellt der Erzähler eine gewisse Nonchalance zur Schau. Vor dem Hintergrund der zeitgenössischen Optik lässt sich zumindest spekulieren, dass die notierten Zahlen mit der Berechnung von Brechungsindizes der Spektralfarben zu tun haben. So könnten z.B. die Dispersionseigenschaften von lichtdurchlässigen Körpern im Hinblick auf die Spektralfarben und deren Eigenschaften und Wirkungen untersucht werden. Einiges davon lässt sich dann im Zusammenhang des Textes quellenmäßig erschließen, wie weiter unten zu zeigen sein wird (5). Zunächst scheinen die relative Zwecklosigkeit des Experiments und die Schädlichkeit des Experimentierens für die Physis des Experimentators im Vordergrund zu stehen.

Denn prompt verspürt Reinhart einen »leise stechenden Schmerz« zuerst im einen, dann im anderen Auge und erkennt dies als Folge seines "anhaltende[n] Treibens" im schroffen Wechsel von Hell und Dunkel. Besorgt darüber, dass diese ödipale Beeinträchtigung "alle[n] sinnliche[n] Forschungen« ein Ende bereiten könnte, sieht er sich "auf ein beschauliches Nachdenken" über sein bisheriges Leben zurückgeführt. An diesem Punkt ist die topische Konstellation bereits etabliert: Das physikalische Experiment mit dem Licht, das mit dem Leben scheinbar nichts zu schaffen hat, wendet sich gegen das existenzielle Leben, das sich beim Experimentieren von dem snatürlichen

$\begin{array}{ll}25 & \text { Ebd., S. } 10 . \\ 26 & \text { Vgl. Rohe: Roman aus Diskursen (wie Anm. 7), S. 143f. } \\ 27 & \text { Vgl. Albrecht Schöne: Goethes Farbentheologie, München } 1987 .\end{array}$ 
Licht abschottet. Die darin angelegte Spannung wird auch die Handlung in Gang setzen: Der Held bricht aus der verdunkelten Studierstube aus, um Licht und Leben in ihrer Konjunktion wiederzufinden bzw. neu zu entdecken und zu ergründen. Diese Konjunktion wird von Lucie personifiziert - von ihrem Oheim und am Ende auch von Reinhart »Lux" gerufen -, während der Name Reinhart als Zusammensetzung aus den beiden Haupteigenschaften des Kristalls gedeutet werden kann. Die Verbindung von Licht und Leben, alliterativ verstärkt und auch als Quasisynonymia lesbar, ist im Gemeinverständnis evident bis zur Banalität. Doch sie figuriert nie als buchstäbliche Formel, sondern erscheint unmittelbar im Spannungsfeld inhärenter, angrenzender oder analoger Polaritäten wie hell und dunkel, Leben und Tod, Schein und Sein, biologischer und sozialer Geschlechtlichkeit, Kultur und Natur oder Zufall und Lenkung, welche die Rahmenerzählung und die Novellen entfalten. ${ }^{28}$

Diesseits und jenseits der asymmetrischen Bedingungsverhältnisse, dass es Licht ohne Leben, aber kein Leben ohne Licht geben kann, befinden sich Licht und Leben also schon zu Beginn in einem Spannungsverhältnis zwischen der anorganischen Wissenschaft der Physik und der physiologisch-existenziellen Lebenspraxis. Das Verhältnis von physischer und moralischer Welt ist denn auch Gegenstand von Reinharts Nachsinnen:

Nachdem er in munterer Bewegung den größten Teil seiner Jugend zugebracht und dabei mit Aufmerksamkeit unter den Menschen genug gesehen hatte, um von der Gesetzmäßigkeit und dem Zusammenhange der moralischen Welt überzeugt zu werden, und wie überall nicht ein Wort fällt, welches nicht Ursache und Wirkung zugleich wäre, wenn auch so gering wie das Säuseln des Grashalms auf einer Wiese, war die Erkundung des Stofflichen und Sinnlichen ihm sein All' und Eines geworden. ${ }^{29}$

In dieser materialistischen Überzeugung hat sich Reinhart von der Menschenwelt und ihren Affekten und Eigenarten abkehren können. Er lacht nur noch über die »Komödien« der »chemischen Stoffe[ ] u und ärgert sich

28 Vgl. Ursula Amrein: Augenkur und Brautschau. Zur diskursiven Logik der Geschlechterdifferenz in Gottfried Kellers "Sinngedicht«, Bern u.a. 1994; Gerhard Neumann: Der Körper des Menschen und die belebte Statue. Zu einer Grundformel in Gottfried Kellers Sinngedicht, in: Mathias Meyer/Gerhard Neumann (Hg.): Pygmalion. Die Belebung des Mythos in der abendländischen Literatur, Freiburg i.Br. 1997, S. 555-591; Hans Dietrich Irmscher: Physik und Liebe. Ein Versuch über Gottfried Kellers "Sinngedicht", in: Peter Ensberg/Jürgen Kost (Hg.): Klassik-Rezeption. Auseinandersetzung mit einer Tradition, Würzburg 2003.

29 Keller: Das Sinngedicht (wie Anm. 22), S. 11. 
über Rechenfehler und zerbrochene Gläser. Die Strukturen und Prinzipien lässt ihn der Erzähler weiterhin theatral imaginieren:

[...] jetzt fühlte er sich nur klug und froh, wenn er bei seiner Arbeit das große Schauspiel mit genoß, welches den unendlichen Reichtum der Erscheinungen unaufhaltsam auf eine einfachste Einheit zurückzuführen scheint, wo es heißt, im Anfang war die Kraft, oder so was. ${ }^{30}$

In wenigen Zügen führt der Text Reinharts naturwissenschaftliches Weltbild auf die Frage nach dem ersten Prinzip zurück und verknüpft sie mit der religiösen, philosophischen und literarischen Tradition, die zuletzt noch der bereits präsente (Goethe'sche) Faust mit dem Anspruch des Universalgelehrten zu beantworten suchte. Zugleich wird mit den chemischen Stoffen angedeutet, dass in Reinharts Laboratorium, wie man bereits aufgrund der Schilderung der Accessoires im Studierzimmer annehmen konnte, durchaus andere naturwissenschaftliche Disziplinen als die Physik praktiziert werden, auch wenn die Physik die Leitwissenschaft darzustellen und damit mechanistische Erklärungsmodelle vorzuherrschen scheinen. Darauf deuten jedenfalls die lässige Behandlung der Frage nach dem Prinzip und die weiteren Ausführungen zur Art des Zusammenhangs der physischen und der moralischen Welt hin, welche die theatrale Bildlichkeit im Marionettenmodus fortsetzt:

Die moralischen Dinge, pflegte er zu sagen, flattern ohnehin gegenwärtig wie ein entfärbter und heruntergekommener Schmetterling in der Luft; aber der Faden, an dem sie flattern, ist gut angebunden und sie werden uns nicht entwischen, wenn sie auch immerfort die größte Lust bezeigen, sich unsichtbar zu machen. ${ }^{31}$

Die Schmetterling-Wendung soll wohl meinen, dass die Erforschung der seelisch-geistigen Welt zeitgenössisch ohnehin nicht hoch im Kurs steht. Gleichzeitig aber scheint Reinharts Wissenschaft den Anspruch zu erheben, diese Phänomene dereinst erklären zu können, da sie an die physische Welt gebunden und also selbst physischer Natur sind. Der Aufschub verweist zurück auf den Kraftbegriff, der somit als terminierter Platzhalter die Leerstelle des Prinzips besetzt. An diesem Punkt werden die bildhaften Gedanken über den inneren Zusammenhang der Dinge abgelöst bzw. überlagert von gestalthaften Bildern der äußeren Welt:

$\begin{array}{ll}30 & \text { Ebd., S. } 11 f . \\ 31 & \text { Ebd., S. } 12 .\end{array}$ 
Jetzt aber war es ihm, wie gesagt, unbehaglich zu Mut geworden; in der Besorgnis um seine Augen stellte er sich alle die guten Dinge vor, welche man mittelst derselben sehen könne, und unvermerkt mischte sich darunter die menschliche Gestalt, und zwar nicht in ihren zerlegbaren Bestandteilen, sondern als Ganzes, wie sie schön und lieblich anzusehen ist und wohllautende Worte hören läßt.

Der »menschlichen Sitte« entwöhnt, weiß er nicht, wie er draußen auf dem »durchsichtige[n] Meer des Lebens" es anstellen sollte, sich der »lieblichen Dinge« zu nähern. Sich impulshaft aus der Bedrückung befreiend, reißt er die Fensterläden auf, eilt in eine "Bodenkammer « hinauf, wo er »in Schränken eine verwahrloste Menge Bücher« findet, »die von halbvergessenen menschlichen Dingen" handeln, um Rat zu finden, was er nun beginnen sollte. Er zieht einen Band heraus und erwischt einen der "Lachmann'schen Lessingausgabe, und zwar de[n], in welchem die Sinngedichte des Friedrich von Logau stehen«. Und wie er das Buch aufschlägt, fällt ihm der Spruch in die Augen: »Wie willst du weiße Lilien zu roten Rosen machen? / Küß eine weiße Galathee: sie wird errötend lachen. «Er bedankt sich beim Autor, ruft: "Welch' ein köstliches Experiment!« und bricht zu Pferd auf. ${ }^{32}$

Die Art und Weise, wie Reinhart vom physikalischen Experiment über die philosophisch-theoretischen Betrachtungen zu dem literarisch inspirierten Experiment kommt, nämlich über eine Serie teils motivierter, teils kontingenter Impulse, führt schon vor, inwiefern die figurenperspektivisch angeführten naturwissenschaftlich-philosophischen Ansätze und Modelle der Komplexität des gelebten Lebens nicht gerecht werden. Angemessen plump lässt der Text Reinhart die Verse von der Verwandlung der weißen Galathee in Rosen durch Küssen auch als plumpe Anwendungsregel verstehen, um ihn dann in den narrativen Entfaltungen des Sinnspruchs eines anderen zu belehren. Die Zufälligkeit, mit der Reinhart auf den Sinnspruch stößt, ruft im Motiv der Brautsuche die eingangs aufgerufene "natürliche Zuchtwahl«, den jüngsten »Gipfel« der Naturwissenschaft, wieder auf: Biologische und sexuelle 'Wahk, Intention und Kontingenz, Wissenschaft und Lebenspraxis werden in ein Spannungsverhältnis gebracht, das die Novellen durchspielen. Die einleitende Rahmenerzählung kommt, eine zyklische Figur vollziehend, wieder in der (anachronistischen) Aktualität der Darwin'schen Evolutionstheorie an, die, wie die oben referierte Forschung zum Grünen Heinrich gezeigt hat, für die Narration maßgebend werden kann. Die dazwischen miterzählte Geschichte der Natur- und Lebenswissenschaften von Newton über die Goethezeit und den Materialismus ist damit nicht

32 Ebd., S. 12 f. 
überwunden oder neutralisiert, sondern bleibt im Raum des Laboratoriums kopräsent. Tatsächlich beziehen sich die verschiedenen Theorien und Modelle ja auch auf verschiedene Sachbereiche, so dass die Darwin'sche Entwicklungstheorie und die materialistische Stoffwechseltheorie einander nicht widersprechen oder historisch ablösen, sondern auch ergänzen und befruchten können. Zudem können den Theorien und Modellen, wie bereits angedeutet, konträre Annahmen über das Wesen der Natur und des Lebens zugrunde liegen.

Die Programmatik der Rahmenerzählung in Bezug auf die Natur- und Lebenswissenschaften besteht mithin nicht darin, die Theorien und Modelle im Sinn eines historischen Prozesses oder gar Progresses darzustellen und zu bewerten, sondern sie als stoffliches Referenzwissen von Natur und Leben in einer Kopräsenz zu verdichten, um es dann narrativ im gelebten Leben zu entfalten. Dabei geht es nicht darum, die lebenspraktische Tauglichkeit der Theorien und Modelle selbst zu verifizieren oder zu falsifizieren; vielmehr werden damit lebensfeindliche Verhaltens-, Handlungs-, Denk- und Fühlweisen kenntlich gemacht, deren 'Fehler` in einem Missverständnis oder Missbrauch des Natur- und Lebenswissens liegt. Dies gilt selbstverständlich nicht nur für das natur- und lebenswissenschaftliche Wissen, sondern ebenso für die kulturellen Modelle und Theorien, wie Gerhard Neumann dies für den von allen Novellen mehr oder weniger stark aktivierten Pygmalionismus überzeugend dargelegt hat. ${ }^{33}$ Ebenso ist es natürlich möglich, bestimmte wissenschaftliche Theoreme oder Modelle als schon breiter und tiefer in die allgemeine Kultur eingegangene Muster wahrzunehmen, wie das Jürgen Rothenberg ausgehend vom Kampf-ums-Dasein-Muster unternommen hat. ${ }^{34}$

Wenn die Rahmenerzählung mit Reinharts Lichtexperiment bereits ein Exempel von lebensfeindlichem Wissenschaftsverständnis statuiert, so bedeutet dies also nicht, dass die dabei artikulierten spezifischen Theoreme und Modelle sowie die philosophischen Begründungsfragen zugunsten eines allgemeinen Kulturwissens an Relevanz verlieren. Sie können, wie die traditionellen Narrative, mit denen sie verknüpft werden, aus der histori-

33 Vgl. Neumann: Der Körper des Menschen und die belebte Statue (wie Anm. 28), insbes. S. $557-573$.

34 Vgl. Rothenberg: Geheimnisvoll schöne Welt (wie Anm. 17), insbes. S. 257-259 u. 274276. 
schen Tiefendimension aktualisiert werden, wie das die zyklische Struktur der Rahmenerzählung und des Novellenkranzes vorführt. ${ }^{35}$

Im Folgenden sollen zunächst einige wichtige Züge der in der Rahmengeschichte verdichteten Vorgeschichte der Natur- und Lebenswissenschaften nachgezeichnet und dann in die für Keller unmittelbare Aktualität des Materialismus eingebettet werden, bevor mit Moleschotts Licht und Leben eine spezifischere Wissensreferenz analysiert und diskutiert wird. Dabei werden der Begriff der Kraft und die merkwürdige Geste, mit der er dargeboten wird, näher eingekreist.

\section{Verdichtete Vorgeschichte der Lebenswissenschaften}

Die von Reinhart aufgeworfene Frage nach dem »Anfang « behauptet, entgegen der Lässigkeit, mit der er sie behandelt, auch diskurshistorisch eine anhaltende Aktualität. Seit den 40er Jahren des 19. Jahrhunderts, angefangen mit dem Materialismusstreit, folgte im deutschsprachigen Denk- und Forschungsraum eine Kontroverse zwischen rationalistisch-materialistischen und vitalistisch-idealistischen Naturauffassungen auf die nächste. Ihre Anlässe waren heterogen, von Entdeckungen im Stoffwechsel über die Erklärung der Entstehung der Arten bis zur Erforschung der neurologischen Vernetzung des Gehirns. Sie gingen mit Verschiebungen und Umbenennungen ineinander über, so dass man auch von einer einzigen längeren Kontroverse in mehreren Etappen sprechen könnte. Einer der Höhepunkte dieser Debatten war die 1872 gehaltene Rede von Emil Du Bois-Reymond Über die Grenzen des Naturerkennens, deren Schlusspunkt er mit der berühmten Behauptung setzte: "Gegenüber dem Rätsel [...], was Materie und Kraft seien, und wie sie zu denken vermögen, muß er [der Naturforscher] ein für allemal zu dem viel schwerer abzugebenden Wahrspruch sich entschließen: ,Ignorabimus. « ${ }^{36}$ Die Provokation gegenüber dem allgemeinen und insbesondere dem naturwissenschaftlichen Fortschrittsglauben, die den sogenannten Ignorabimus-

35 Schon früh hat Keller dem teleologischen Fortschrittsdenken ein von den narrativen Stoffen her gedachtes zyklisches Modell entgegengesetzt; vgl. Keller: Brief an Hermann Hettner, Berlin, 26. Juni 1854, Bd. 1 (wie Anm. 14), S. 396-401.

36 Emil du Bois-Reymond: Über die Grenzen des Naturerkennens. In der zweiten allgemeinen Sitzung der 45. Versammlung Deutscher Naturforscher und Ärzte zu Leipzig am 14. August 1872 gehaltener Vortrag, in: Ders.: Vorträge über Philosophie und Gesellschaft, eingeleitet und mit erklärenden Anmerkungen hg. von Siegfried Wollgast, Hamburg 1974, S. 54-77, hier S. 77. 
Streit auslöste, bestand im Futur des Nichtwissens. Statt mit Materie und Kraft hätte Du Bois-Reymond das Rätsel auch mit anderen Begriffen formulieren können: Körper (oder Leib) und Seele, Materie und Geist, Stoff und Kraft. Die Kombination quer durch die konventionellen Dualismen indiziert die längerfristige Dimension des >Wahrspruchs‘, vermeidet aber den Begriff des Lebens, der sich auch damals als Resultante der verwendeten Größen aufdrängte. Der zeitlich näher liegende Grund für diese Vermeidung liegt wohl darin, dass die erst ein gutes Jahrzehnt alte Verkündigung der Entstehung der Arten just als Lösung des Rätsels des Lebens hoch im Kurs stand, obwohl Darwin snur die Entwicklung der Lebensformen und -funktionen durch Selektion erklärte und keineswegs den Ursprung und das Wesen des Lebens, von der chemisch-physiologischen Erzeugung bis zum elementaren Selbstgefühl des Lebewesens, geschweige denn den $\mathrm{Zu}-$ sammenhang dieser Größen, also das, was Du Bois-Reymond mit Materie, Kraft und Denken meinte.

Ein tieferer Grund für die Vermeidung des Lebensbegriffs könnte die Nachwirkung der naturphilosophisch-romantischen Beanspruchung des 'Lebens sein, das als vitalistisches Prinzip die Systemstelle Gottes besetzte. ${ }^{37}$ Anatomie und Physiologie bauten nach 1800 auf die Konzeption des Organismus, die Immanuel Kant 1790 in der Kritik der teleologischen Urteilskraft in Auseinandersetzung mit den zeitgenössischen Forschungen formuliert hatte: Hier rückt und stutzt Kant den Begriff des Zwecks bzw. der Endursachen erkenntniskritisch als regulatives Prinzip zurecht: "Ein organisiertes Produkt der Natur ist das, in welchem alles Zweck und wechselseitig auch Mittel ist. . ${ }^{38}$ Die Kraft der Hervorbringung eines Organismus erklärt er für prinzipiell »unerforschlich[ ] «, nennt sie jedoch in Bezug auf ihren Zweck mit Johann Friedrich Blumenbach »Bildungstrieb ${ }^{39}{ }^{39}$ Den Lebensbegriff eskamotiert er explizit, obwohl er der »unerforschlichen Eigenschaft« der organisierten Materie nahe komme; denn dadurch würde man der Materie entweder ein immaterielles Prinzip, also eine Seele oder einen Geist, beigesellen oder dann die Materie insgesamt als belebt annehmen müssen. ${ }^{40}$ Zwar kann »schlechter-

37 Vgl. Friedrich Albert Lange: Geschichte des Materialismus und Kritik seiner Bedeutung in der Gegenwart [1866], wohlfeile Ausgabe, besorgt und mit biographischen Vorwort versehen von Hermann Cohen, Iserlohn/Leipzig 1886, S. 453f., und Ajouri: Erzählen nach Darwin (wie Anm. 4), S. 64-143.

38 Immanuel Kant: Kritik der Urteilskraft [1790], § 66, in: Ders.: Werkausgabe, zwölf Bände, hg. von Wilhelm Weischedel, Frankfurt a.M. 1977, Bd. 10, S. 324.

39 Ebd., § 81, S. 381; vgl. Johann Friedrich Blumenbach: Über den Bildungstrieb und das Zeugungsgeschäfte, Göttingen 1781.

40 Kant: Kritik der Urteilskraft (wie Anm. 38), § 65, S. 321-323. 
dings keine menschliche Vernunft [...] die Erzeugung auch eines Gräschens aus bloß mechanischen Ursachen zu verstehen hoffen", es sei aber aufgrund der Beschaffenheit der Erkenntnis angezeigt, »alle Produkte und Ereignisse der Natur, selbst die zweckmäßigsten, so weit mechanisch zu erklären, als es immer in unserm Vermögen [...] steht «. ${ }^{41}$

Der Begriff des Lebens hatte jedoch in der Naturwissenschaft und Philosophie zwischen 1750 und 1800 eine ganze Diskursrevolution mit bewegt. Für La Mettrie und Diderot, aber dann vor allem für den empirisch orientierten Pflanzenkundler und Physiologen Caspar Friedrich Wolff stand der Lebensbegriff im Zentrum ihrer Kritik an der dualistisch-mechanistisch begründeten Konzeption der von Albrecht von Haller vertretenen Präformation. Wolff, der mit allem Recht als erster Biologe im modernen Sinn gelten kann, vermochte Schritt für Schritt am Material aufzuzeigen, dass organische Körper, ob Pflanze oder Tier (oder Mensch), ihre Gestalt und Größe nicht aufgrund der immer schon fix vorgeformten Materie erhalten, die sie zum Wachsen lediglich auszuwickeln brauchen, sondern durch Formung und Vermehrung kleinerer und weniger gestalteter Grundelemente selbst herausbilden. Als »Prinzip« der »Generation«, wie er den Prozess im Sinn von Erzeugung und Entstehung nannte, nahm Wolff eine "wesentliche Kraft" an, ohne dass er deren Verbindung mit der Materie näher bestimmte. ${ }^{42}$ Die Art der Erzeugung und des Wachstums sowie diese wesentliche Kraft seien die Eigentümlichkeiten, "weswegen wir ihnen", diesen Körpern, »ein Leben zuschreiben«, und dies habe nichts mit den Gebilden der Präformation zu tun. ${ }^{43}$

Die in der Theoria generationis 1759, genau hundert Jahre vor Darwins Entstehung der Arten, aufgestellten Hypothesen einer Lebenskraft (unter dem Begriff der vis essentialis) und der epigenetischen Entwicklung haben es überhaupt erst erlaubt, das Leben als eine eigene dritte Größe, die Körper und Seele integriert, und eine von der Physik abgegrenzte Wissenschaft des Lebens und des Lebendigen zu etablieren. Zahlreiche Autoren (Medicus, Blumenbach, Brandis, Humboldt, Reil, Roose, Treviranus) mit mehr oder weniger aktueller und historischer Wirkung entwarfen von 1770 bis 1800 im deut-

41 Ebd., $\$ 78$, S. 370f.

42 Caspar Friedrich Wolff: Theoria generationis [1759] / Über die Entwicklung der Pflanzen und Thiere, I., II. und III. Theil, übers. und hg. von Paul Samassa, Leipzig 1896, Nachdruck mit einer Einleitung von Olaf Breidbach, Thun 21999, S. 4-10.

43 Caspar Friedrich Wolff: Theorie von der Generation in zwo Abhandlungen erklärt und bewiesen von Caspar Friedrich Wolff, der Arzneygelahrtheit Doktor, Berlin 1764, Nachdruck: Hildesheim 1966, S. 160. 
schen Sprachraum biologische Konzeptionen des Lebens zwischen einer Organismustheorie, deren Strukturen kausal-mechanisch gedacht waren, und einer Lebenskrafttheorie, die letztlich metaphysisch begründet war. Während Theodor Georg August Roose 1797 in Grundzüge der Lehre von der Lebenskraft diese >Wissenschaft vom Leben< erstmals unter dem Begriff »einer Biologie« ${ }^{44}$ formulierte, welche die Lebenskraft ins Zentrum stellte, löste Gottfried Reinhold Treviranus fünf Jahre später seine Biologie oder Philosophie der lebenden Natur bereits in verschiedene Teilsysteme auf, ein materialistisches, ein vitalistisches und ein organistisches System, eine Unterteilung, die auf die nachfolgende Ausdifferenzierung der naturwissenschaftlichen Disziplinen verweist. ${ }^{45}$

Wenn das Leben sich seitdem als vielgestaltiger und zugleich totaler Begriff erhalten hat, dann zum einen deswegen, weil er parallel und in mehr oder weniger dichter Vernetzung mit Wissenschaft und Philosophie zunehmend zur Zentralreferenz des politischen, ökonomischen und sozialen Denkens und Handelns und dabei Gegenstand dessen geworden ist, was Michel Foucault und Giorgio Agamben unter dem Titel der Biopolitik dargelegt haben. Zum anderen hat sich das Verhältnis von Literatur und Leben gewandelt: Literatur - und Kunst allgemein - beansprucht nicht bloß, das Leben darzustellen, sondern das Leben zu sein, in einem substanziellen und energetischen Sinn. Goethes Werther ist die Manifestation dieser Engführung und die Performanz der Aporie, die den Protagonisten, aber auch den Sturmund-Drang-Autor Jakob Michael Reinhold Lenz verschlungen hat. Doch seither ist das Bestreben, die reine energetische Intensität darzustellen bzw. vorzuführen, der Literatur als defigurierende Kraft eingeschrieben, so sehr die Literatur auch immer Nachahmung von Gestalt geblieben ist. Goethe, der mit Lenz selbst zu den Entfesslern der literarischen Lebenskraft gehört, hat die Aporie der Un/Darstellbarkeit in der Dialektik von Kraft und Gestalt aufgelöst: Das Leben kann sich nicht von der Natur lossagen, sondern nur seine Kraft, die eine Naturkraft ist, möglichst lang zu Bildung, Wachstum und Erhaltung seiner ideal bestimmten Gestalt und inneren Strukturen einsetzen. Im Goethe'schen Verständnis bildet das Leben für den mit Selbstbewusstsein und Willen ausgestatteten Menschen eine notwendige, wenn auch illusorische (weil provisorische) Ausnahme von den unerbittlichen Gesetzen der verschlingenden Natur. Diese »lebensnotwendige Illusion< einer

44 Theodor Georg August Roose: Grundzüge der Lehre von der Lebenskraft [1797], 2., verbesserte Aufl., Göttingen/Braunschweig 1800, S. III.

45 Gottfried Reinhold Treviranus: Biologie oder Philosophie der lebenden Natur für Naturforscher und Ärzte, sechs Bände, Bd. 1, Göttingen 1802, S. 83-103. 
(relativen) Autonomie steht in einem doppelten Bedingungsverhältnis: zum einen mit der objektivierbaren Ablösung der tierischen Triebe vom Naturgebrauch und ihrer Sublimierung in shöhere Vermögen und sfeie‘ Künste, zum anderen mit der subjektiven Perspektive der menschlichen Wahrnehmung, Empfindung und Erfahrung, die sich in der emphatischen Setzung der (eigenen) Gestalt manifestiert.

Im ästhetischen Denken und Schaffen öffnet sich ein mehrfach ausgerichteter Antagonismus, der sich bis zur Kluft vertiefen kann: zwischen der immanenten Begründung der Kunst im und als Natur-Leben einerseits und den künstlich-medialen Ausdrucksmöglichkeiten andererseits; zwischen der energetischen Emanation einerseits und der mechanischen Artikulation andererseits, die immer in die Regelrhetorik zurückzufallen droht; zwischen der gesetzmäßigen Entwicklung des Organismus von innen heraus einerseits und der subjektiven Gestaltung von außen andererseits. Kant vermochte mit seiner Kritik der Urteilskraft den Antagonismus nicht aufzulösen, sondern konsolidierte ihn in der Trennung zwischen der ästhetischen und der teleologischen Urteilskraft: Während der Lebensbegriff wegen seiner transzendenzträchtigen Ambivalenz aus der objektiven Erkenntnis der teleologischen Urteilskraft ausgeschlossen wird, schließt ihn die ästhetische Urteilskraft in die subjektive Erkenntnis ein, und zwar als sogenanntes Lebensgefühl: Es ist die Bilanz von Lust und Unlust des freien Spiels der Erkenntniskräfte, die sich im Gemüt als Lebensgefühl manifestiert. ${ }^{46}$

Johann Gottfried Herder hat diesen Ausschluss/Einschluss-Mechanismus in Bezug auf das Leben in Kants Philosophie und als grundlegendes Problem nicht nur der Ästhetik schon früh erkannt. ${ }^{47}$ Weniger explizit und ausführlich, aber dafür pointiert hat dann im 19. Jahrhundert Georg Büchner die Kluft zwischen der objektiven Naturerkenntnis, die mit der Organismustheorie das strukturelle Modell der Ästhetik lieferte, und der subjektiven vitalen Empfindung als ästhetischem Kernelement ausgelotet. Der Riss, der sich in einem weiten Hyperbaton zu Beginn von Büchners Erzähltext Lenz (1835) in der Brust des Protagonisten öffnet, ist die poetische Artikulation desselben Problems, das er in der Probevorlesung über Schädeherven (1837) auf

46 Vgl. Kant: Kritik der Urteilskraft (wie Anm. 38), § 1, S. $115 f$.

47 Vgl. Hubert Thüring: Das neue Leben. Studien zu Literatur und Biopolitik 1750-1938, München 2012, S. 111-122; Ders.: Leben, in: Büchner-Handbuch, hg. von Roland Borgards und Harald Neumeyer, Stuttgart/Weimar 2009, S. 209-217, und Ders.: Sprache als Rhetorik des Lebens. Zu den Anfängen einer fundamentalen Differenz von Herder und Kant, in: Ralf Simon (Hg.): Herders Rhetoriken im Kontext des 18. Jahrhunderts, Heidelberg 2014, S. 157-173. 
erkenntnisphilosophischem Terrain zu formulieren sucht. Dass der Weg zwischen Erkennen und Empfinden, Struktur und Energie, Gestalt und Kraft usw. als Phänomene des Lebens nur poetisch begangen werden kann, wird von Büchner nirgendwo programmatisch verkündet, sondern von und in den literarischen Texten performiert und reflektiert.

Die Literatur wird um 1800 zum privilegierten Ort der Erkenntnis des Lebenswissens im Spannungsfeld der alten und neuen fundamentalen Dichotomien und der wissenschaftlich-disziplinären Ausdifferenzierung. Denn die naturwissenschaftlichen Disziplinen erhoben Kants erkenntnismethodisches Regulativ einer möglichst weitgehenden mechanischen Erklärung der Phänomene zum Grundsatz von Wissenschaftlichkeit, ohne ihre transzendentale Bedingtheit zu reflektieren, während der von Schelling inspirierte Idealismus an einem fundamentalen Lebensprinzip festhielt, ohne wissenschaftliche Operabilität zu bedenken. Neben einem Mangel an Reflexion der gemeinsamen Grundbedingungen fehlten zudem weitgehend die Bezüge zur Lebenspraxis und zur Ästhetik, die in der Literatur im Vordergrund stehen. Doch vermögen literarische Texte die philosophischen und wissenschaftlichen Bedingungen und Verfahren in Bezug auf ihre eigenen mit zu inszenieren und zu reflektieren. In Analogie zu den Tendenzen der Wissenschaften können auch in der Literatur Tendenzen zwischen einer vitalistischen Defiguration, welche die Texte an die Grenzen der Darstellbarkeit treibt, und einer organologischen (Re)Figuration, die sie an die (aristotelische) Darstellungspoetik zurückbindet, beobachtet werden.

\section{Kellers Poetik des Lebens in Auseinandersetzung mit dem Materialismus Feuerbachs und Moleschotts}

Kellers Texte würde man wohl ohne Zögern der (re)figurierenden Tendenz zuordnen, doch lassen sich defigurierende Momente ausmachen, die auch untergründig fortwirken. Dass und wie Kellers Texte unter dem Einfluss des Materialismus und der Evolutionstheorie die narrative Teleologie auflösen und stattdessen die innere Vernetzung verdichten und sich gleichzeitig durch die explizit inszenierte Zufälligkeit öffnen, ist bereits dargelegt worden. Dies lässt sich noch weitgehend auf die Referenz einer Natur beziehen, die zugleich gesetzmäßig und, durch ihre Komplexität, geheimnisvoll wirkt, wie Keller nach der frühen Erfahrung des Materialismus schreibt. In der zweiten Fassung des Grünen Heinrich erscheint die generalisierte Natur dann auch als Feindin des Lebens. Doch erst im Sinngedicht wird deutlich, 
inwiefern jede Vorstellung von strikter Naturgesetzlichkeit unvermeidlich reduktiv ist und sich in der bewussten oder unbewussten Anwendung auf das humanisierte und kulturalisierte Leben schädlich oder tödlich auswirkt. Die Eröffnung der Rahmenerzählung führt dies, indem sie selbst reduktiv agiert, durch die Kurzschließung von physikalischer Naturauffassung mit der Gefährdung der Gesundheit im Experiment exemplarisch vor. Auch die Reduktion wird (im Sinn von 'Rückführung`) buchstäblich vorgeführt, indem Reinhart theoretisch den "unendlichen Reichtum der Erscheinungen unaufhaltsam auf eine einfachste Einheit« zurückgehen sieht, "wo es heißt, im Anfang war die Kraft, oder so was «. ${ }^{48}$ Dieses Moment der Unbestimmtheit erscheint ironisiert, aber die Ironie ist nicht einfach aufzulösen: Drückt Reinhart - und mit ihm solidarisch der Erzähler - damit seine Überlegenheit gegenüber Ursprungs- und Begründungsfragen aus, wie sie (faustische) Theologen und Philosophen von gestern und heute stellen, während sie für seine positive Wissenschaft und sein Weltverständnis belanglos sind? Oder lässt der Erzähler ihn entweder als ignorant und überheblich gegenüber einer für unumgänglich erachteten Frage erscheinen oder als naiv, weil die Annahme einer ursprünglichen Kraft einen Transzendenzglauben impliziert? Explizit löst der Text die Unbestimmtheit nicht auf, so dass man diese Unbestimmtheit selbst als Aussagegehalt annehmen muss. Implizit, und das heißt hier narrativ, erscheint dieses Moment der Unbestimmtheit als Wendepunkt, nach dem sich Reinhart wieder den "moralischen Dinge[n]《 und dem sinnlichen Leben, zunächst in literarischer, dann in realer Form, zuwendet. Es ist, wie wenn der Kraftbegriff nicht nicht getrennt werden kann, damit Reinhart und der Erzähler das (erzählte) Leben und das (gelebte) Erzählen beginnen können. Es ist der Punkt, an dem die Erzählung ein Moment vitalistischer Defiguration frei - und sich dem Inkalkulablen aussetzt. Die Frage nach dem Verhältnis von Kraft (oder Geist) und Materie, Stoff oder Form, die nach 1800 mit Kants pragmatisch-operativer Hypothese und der idealistisch-romantischen Verabsolutierung zwei vorderhand wenig miteinander kommunizierende Antworten gefunden hatte, gewann im Zug neuer Erkenntnisse und Entdeckungen um 1830 neu an Virulenz, die sich im Materialismusstreit, in den Debatten um die Selektionstheorie und im Ignorabimus-Streit artikulierte. Zur mutmaßlichen Zeit des Geschehens der Rahmenhandlung (nach 1850), in die auch die Entstehung von Teilen des Sinngedichts zurückreicht, und auch zur Zeit der hauptsächlichen Arbeit und Veröffentlichung hatte die Frage eine sowohl allgemeine als auch spezifische

${ }^{48}$ Keller: Das Sinngedicht (wie Anm. 22), S. 12. 
Aktualität. In Das Wesen der Religion stellt Feuerbach die mit Das Wesen des Christentums (1841) lancierte Religionskritik auf eine naturwissenschaftliche Basis und versucht dabei die besondere Stellung des Menschen in der Natur zu bestimmen: Einerseits ist der Mensch ein Naturwesen, lebt »in der Natur, mit der Natur, von der Natur « - eine Wendung, an welche die oben zitierte Stelle aus Kellers Briefen anklingt ${ }^{49}$-, andererseits erscheint er als »ein von der Natur unterschiedenes [...] und auf sich selbst sich konzentrierendes Wesen ${ }^{50}{ }^{50}$ Der durch das Bewusstsein bedingte Abstand des Menschen zur Natur bedingt seinerseits sein Nichtwissen über das Wesen des Lebens. Die "Unerklärlichkeit des organischen, insbesondere menschlichen Lebens aus der Natur« des Lebens haben die Menschen mit immateriell-göttlichen Ursachen ausgefüllt. ${ }^{51}$ Die Unerklärlichkeit hält Feuerbach - anders als Kant oder Du Bois-Reymond - für eine "relativ-subjektive« und auch provisorische, ${ }^{52}$ denn ansonsten bliebe der Begriff des Lebens ein Einfallstor der Transzendenz. Im Sinn eines relativen Fortschritts begrüßt er neue wissenschaftliche Forschungen, welche die Lücke des Lebenswissens zwischen den abstrakten Größen `Natur und `Mensch w weiter physiologisch zu vermitteln vermögen, insbesondere die Forschungen zum Stoffwechsel.

In seinem Aufsatz Die Naturwissenschaft und die Revolution von 1850, der hauptsächlich Jakob Moleschotts frisch erschienene Lehre der Nahrungsmittel. Für das Volk (1850) rezensiert, ${ }^{53}$ führt Feuerbach zunächst die Erkenntnisse der Naturwissenschaft von der Einheit und relativen Gleichheit der Naturwesen gegen die "Anmaßungen und Fiktionen " ${ }^{54}$ der politischen und religiösen Unterdrückung ins Feld und zeigt das revolutionäre Potenzial der Naturwissenschaft am Beispiel von Kopernikus auf, der als einer der Ersten mit seiner Schrift das "ganze Glaubenssystem der alten Welt erschüttert« hat. Das aktuelle Beispiel liefert sodann Moleschotts frisch erschienene "revolutionäre Schrif $«{ }^{55}$ Darin findet er die »schwierigsten Probleme der Philosophie gelöst«, nämlich die »Frage vom Bande zwischen dem Leib und der Seele«:

49 Ludwig Feuerbach: Das Wesen der Religion [1841], § 16, in: Ders.: Gesammelte Werke (wie Anm. 20), Bd. 10, S. 3-121, S. 19.

50 Ebd. $\$ 37$, S. 43.

51 Ebd. $§ 15$, S. 18, vgl. $§ 23$, S. 26f. und S. 81 (Ergänzungen und Erläuterungen zum »Wesen der Religion«: "Die Existenz, das Leben ist das höchste Gut, das höchste Wesen - der ursprüngliche Gott des Menschen."

52 Ebd., $\$ 15$, S. 18.

53 Jakob Moleschott: Lehre der Nahrungsmittel. Für das Volk, Erlangen 1850.

54 Ludwig Feuerbach: Die Naturwissenschaft und die Revolution [1850], in: Ders.: Gesammelte Werke (wie Anm. 20), Bd. 10, S. 347-368, hier S. 351.

55 Ebd., S. 356. 
»Jetzt wissen wir aus wissenschaftlichen Gründen, was längst das Volk aus der Erfahrung wusste, dass Essen und Trinken Leib und Seele zusammenhält, dass das gesuchte Band also die Nahrung ist «. ${ }^{56}$

Mit Moleschotts Forschungen betrachtet er nicht nur die »Identität von Geist und Natur« als erwiesen, sondern auch die Qualität des Daseins und des Denkens: »An das phosphorhaltige Fett ist die Entstehung, folglich auch die Tätigkeit des Hirns geknüpft ... Ohne Phosphor kein Gedanke,${ }^{57}$ so zitiert er aus der Lehre der Nahrungsmittel einen im Materialismusstreit zum Schlagwort geronnenen Befund und nennt mit Fleisch und Hülsenfrüchten auch die entsprechenden Lieferanten. ${ }^{58}$ Feuerbach erhebt die Nahrung zum »Spino-

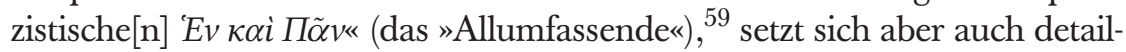
lierter mit Moleschotts Ausführungen zu den "chemischen Grundstoffe[n] oder Elemente[n] der Nahrungsstoffe«, den physiologischen Ernährungsund Stoffwechselprozessen sowie den Folgen des Fastens und Hungerns auseinander. Die Einsicht, dass man dem Volk, wenn man es bessern will, "statt Deklamationen gegen die Sünde bessere Speisen« geben soll, prägt er zur berühmt gewordenen Formel: »Der Mensch ist, was er ißt«. ${ }^{60}$

Feuerbach schlägt mithin weite Brücken zwischen einzelnen chemischen und physiologischen Modellen, anthropologischen Bestimmungen sowie den religiösen und politischen Ideologien und Verhältnissen. Die offensichtliche Begeisterung für Moleschotts Stoffwechselerkenntnisse dürfte sich, wie bereits erwähnt, an den Aufschlüssen über das "Band zwischen Leib und Seele«, d.h. über das sunerklärlicher Leben, entzündet haben. Tatsächlich zitiert er die entsprechende Emphase Moleschotts auch in emphatischer Weise: "Das Leben ist Stoffwechsel. ${ }^{61}$ Diese Perspektive verspricht, die Verbindung zwischen den chemisch-physiologischen Prozessen von Aufnahme, Verarbeitung und Aussonderung von Stoffen des einzelnen Orga-

56 Ebd., S. 357.

57 Ebd., S. 359f.

58 Vgl. ebd., S. 363-367. Die wissenschaftliche Erforschung der Nahrungsmittel zu verschiedenen, teils unmittelbar biopolitischen Zwecken hat in Bezug auf den in Hülsenfrüchten vorhandenen »Erbsenstoff» mit Georg Büchners Woyzeck (1837/38) literarische bzw. literatur- und kulturwissenschaftliche Prominenz erlangt; vgl. stellvertretend Harald Neumeyer: "Hat er schon seine Erbsen gegessen? Georg Büchners >Woyzeck Ernährungsexperimente im ersten Drittel des 19. Jahrhunderts«, in: Deutsche Vierteljahrsschrift für Literaturwissenschaft und Geisteswissenschaft 83 (2009), 218-245.

59 Feuerbach: Die Naturwissenschaft und die Revolution (wie Anm. 54), S. 358.

60 Ebd., S. 367.

61 Moleschott: Lehre der Nahrungsmittel (wie Anm. 53), S. 60, zit. in: Feuerbach: Die Naturwissenschaft und die Revolution (wie Anm. 54), S. 360. 
nismus einerseits und den Prozessen von Leben und Tod im Kreislauf der organischen und anorganischen Natur andererseits zu erhellen.

Tatsächlich wird Moleschott den Lebensbegriff fortan programmatisch bearbeiten, wie neben Licht und Leben (1856) sein Hauptwerk Der Kreislauf des Lebens (1852), der Vortrag über Die Einheit des Lebens (1864) und weitere Schriften zeigen. Ziel ist es, den Menschen als Lebewesen neben anderen einzureihen und die Phänomene des Lebens möglichst in der »Naturnothwendigkeit« aufgehen zu lassen. ${ }^{62}$ Das Leben wird dabei zum Inbegriff der "Natureinheit« von »Kraft und Stoff, Geist und Mensch, Naturgesetz und Weltall« und der analogen Dichotomien, deren Glieder nicht voneinander ableitbar seien. ${ }^{63}$ So sehr Feuerbach die wissenschaftlichen Befunde und Argumente Moleschotts begrüßt und beansprucht, möchte er doch den Umständen, dass die begriffliche Unterscheidung von Materie und Geist im Leben des Menschen eine phänomenale Grundlage aufweist und dass das Denken dem Menschen einen analytischen und reflektierenden Abstand zur äußeren Natur ebenso wie zu seiner eigenen Natur ermöglicht, Rechnung tragen. Seine Philosophie bleibt denn auch nach der episodischen Feier des (populär)wissenschaftlichen Materialismus anthropologisch ausgerichtet: Das »bewußtlose Wesen der Natur« ist ihm das "erste Wesen [...] der Zeit, nicht dem Rang nach, das physisch, aber nicht moralisch erste Wesen; das bewußte menschliche Wesen ist [ihm] das [...] dem Range nach erste Wesen ${ }^{64}{ }^{6}$ Für Feuerbach erscheint das Leben auch (oder erst) in der Perspektive des Stoffwechsels als das unbestimmte Dazwischen von Natur und Geist, als Medium, aus dem der Mensch lebend nicht heraustreten kann, während Moleschott das Leben auf das ganze Naturgeschehen ausweitet und den Menschen darin aufgehen lässt:

Die ersten Ringe in der Kette des Thierlebens verschlingen sich mit den Trieben jener organisirenden Schöpferkraft, welche die Pflanzen als das blühende Reich der unbewußten Dichtung erscheinen läßt. Durch die Thätigkeit des Sauerstoffs wird das Blut theilweise gebildet von dem Träger der Feuerglut, der es läutert zu dem Gewebe, dessen Stoffwechsel die Gedanken bedingt, der aber auch Hirn und Blut wieder verbrennt zu den einfachen Verbindungen, aus denen sich die knospende Pflanze verjüngt. Es ist Tod in dem Leben und Leben im Tode. Dieser Tod ist kein schwarzer, schreckender. Denn in der Luft und im Moder schweben und ruhen

62 Jacob Moleschott: Licht und Leben. Rede beim Antritt des öffentlichen Lehramtes zur Erforschung der Natur des Menschen an der Zürcher Hochschule. Gesprochen am 21. Juni 1856, Frankfurt a.M. 21856, S. 27.

63 Ebd., S. 32-34.

64 Ludwig Feuerbach: Vorlesungen über das Wesen der Religion [1851], in: Gesammelte Werke (wie Anm. 20), Bd. 6, S. 29. 
die ewig schwellenden Keime der Blüthe. Wer den Tod in diesem Zusammenhang kennt, der hat des Lebens unerschöpfliche Triebkraft erfaßt und mit ihr die ganze Fülle der menschlichen Dichtung, die unwandelbar ruht auf den Marmorsäulen der Wahrheit. ${ }^{65}$

Die chemischen und physiologischen Prozesse werden direkt mit den Universalbegriffen von Leben und Tod gekoppelt, und die Unanschaulichkeit beider Komplexe wird mittels Tropen als poetische Schöpfung des Lebens selbst überhöht. Die menschliche Perspektive des Handelns, Denkens und Fühlens als erlebte Sphäre des Lebens, die Feuerbachs religiöse und politische Kritik einbezieht, wird nicht problematisiert, sondern ihrerseits allegorisch vom Wahrheitspathos untermauert.

Kellers persönliche Kenntnis von Moleschotts und Feuerbachs Schriften, insbesondere jener zur Ernährung und zum Stoffwechsel, ist bekannt, ${ }^{66}$ und die Bedeutung dieser Erkenntnisse für Kellers Erzählen ist zum Teil eingehender erforscht worden. Neben der ausführlichen Hungerepisode des Protagonisten und anderen kulinarischen Szenen im Grünen Heinrich findet sich das Ernährungsmotiv auch in den Seldwyla-Novellen Pankraz, der Schmoller und Spiegel, das Kätzchen (1856) sowie in den Sinngedicht-Novellen Die arme Baronin oder auch Regine im Sinn einer - mehr oder weniger humoristisch gebrochenen - materialistischen Welt- und Lebensauffassung und mit teils deutlichen Quellenbezügen narrativ ausgestaltet. ${ }^{67}$ Dies- und jenseits der grundsätzlichen Zustimmungen lassen sich schon in den expliziteren Briefstellen sowie den Erzähler- oder Figurenreden, die oben im Forschungsbericht angeführt worden sind, jene Differenzierungen des Lebensbegriffs gegenüber dem Naturbegriff herausarbeiten, die sich in Feuerbachs eigenen Argumentationen und in Differenz zu Moleschott haben akzentuieren las-

65 Jacob Moleschott: Der Kreislauf des Lebens. Physiologische Antworten auf Liebig's chemische Briefe [1852], Mainz 21855, S. 455.

66 Vgl. Keller: Brief an Johanna Kapp, Heidelberg, 7. und 11. Dezember 1849, Gesammelte Briefe (wie Anm. 14), Bd. 2, S. 28: "Herr Moleschott las einen Abschnitt aus einem diätetischen Werke vor, das er schreibt, das Kapitel über Hunger und Durst. Es kam darauf hinaus, daß man sterben müsse, wenn man Nichts mehr esse und trinke, was mich sehr frappirte. Allerlei häßliche physiologische Ausdrücke trug er, um die Pille zu vergolden, mit einer Sorte von süßem Pathos vor, welche mir, trotz meines Elendes, einen abscheulichen Lachkrampf verursachte, was mir fast übel bekam." Vgl. auch Keller: Brief an Hermann Hettner, 17. Februar 1851, Bd. 1 (wie Anm. 14), S. 389, wo Keller von seinem »Vergnügen« bei der Lektüre von "Feuerbachs Aufsatz über sein [Moleschotts] Buch« berichtet.

67 Vgl. Rohe: Roman aus Diskursen (wie Anm. 7), S. 162-171, zum Grünen Heinrich und zu Spiegel, das Kätzchen; zu den anderen Texten liegen meines Wissens noch keine eingehenden quellen- und diskursbezogenen Forschungen vor. 
sen. Aber erst die Rahmenerzählung des Sinngedichts bringt diese zu jener nuancierten Darstellung, die bereits ausgelegt worden ist. Es lässt sich nun zeigen, dass dies bei aller Vielfalt und Dichte in einer teils fast unmittelbaren Auseinandersetzung mit Moleschotts Licht und Leben geschieht.

\section{Licht und Leben}

An der neuralgischen Stelle, wo Reinhart seine Natur- und Weltauffassung rekapituliert und mit der lässig erscheinenden Wortgeste auf den biblisch-faustischen Topos zurückführt - »im Anfang war die Kraft, oder so was« -, verweist der Kommentar der historisch-kritischen Keller-Ausgabe auf Moleschotts Licht und Leben und zitiert daraus den folgenden Passus:

Kraft und Stoff, sind nichts Anderes als verschiedene Anschauungsweisen für Ein und dasselbe Ding, man mus bei dem Ausdruck Kraft zugleich immer an den Stoff, aber ebenso auch bei dem Worte Stoff jederzeit an die Kraft denken. Die Materialisten sagen mit Faust: »im Anfang war die That. ${ }^{68}$

Vom Wortbegriff her scheint sich Reinhart damit markant gegen das materialistische Axiom der Einheit zu positionieren. Wie lässt sich diese Abweichung in Bezug auf die oben formulierten Fragen ausdeuten? Moleschott erklärt es für ein "grobes Missverständnis, das aller Orten begangen wird, wenn man der stofflichen Anschauung von Kraft die Absicht unterlegt, durch den Hinweis auf jene wesenhafte Verschmelzung des Stoffes und der Kraft den Geist oder das Leben zu erklären.« Die »Verknüpfung« von Leib und Geist sei keine "Erklärung", sondern eine "Thatsache" ebenso wie die physikalische "Schwerkraft«, die "aus der Masse erklären zu wollen« sich auch »kein Denker« einrede. ${ }^{69}$ Durch den Vergleich mit der anorganischen Physik kassiert Moleschott die Differenz zum Leben, das er durch die Bedeutung des Lichts eigentlich als ein besonderes Phänomen zu erhellen verspricht. Das entspricht konsequent dem schon im Kreislauf des Lebens exponierten Bestreben, das Rätsel des Lebens als solches aus der

${ }^{68}$ Moleschott: Licht und Leben (wie Anm. 62), S. 32f., hier zit. nach der ersten Auflage und dem Keller gewidmeten Exemplar der Zentralbibliothek Zürich; vgl. Kommentar zum Sinngedicht, HKKA 23.1, S. 283. Im Weiteren wird wieder wie oben aus der zweiten Auflage zitiert, die einige Textänderungen aufweist; diese sind für die Argumentation hier nicht wesentlich.

${ }^{69}$ Moleschott: Licht und Leben (wie Anm. 62), S. 33f. 
Welt zu schaffen, indem die Differenz zwischen dem Anorganischen und Organischen durch die Ausweitung des Lebensbegriffs überspielt wird. Die Abweichung im Wortbegriff ist mithin sachlich motiviert, was auf eine fundamentale Differenz und programmatische Absetzung, wie oben bereits als These skizziert, schließen lässt. Dennoch bleibt die rhetorische Geste der Beliebigkeit noch deutungsbedürftig. Bevor hier noch einmal anzusetzen ist, sollen noch ein paar Referenzlinien und -punkte aus Licht und Leben angeführt und kommentiert werden.

Moleschotts Zürcher Antrittsvorlesung entwickelt den Zusammenhang von Licht und Leben von den elementarsten Lebensformen bis zum Menschen und von den chemischen Prozessen bis zum großen organisch-anorganischen Kreislauf anhand des elementaren Stoffwechsels von Sauerstoff, Kohlendioxid und Wasser. Ausgangspunkt ist die einfache experimentelle Beobachtung, dass sich in einer mit »Brunnenwasser « gefüllten »Flasche von weißem Glase" am »Licht" alsbald grünlicher sogenannter "Priestley'sche[r] Schleim» bildet, bestehend aus organisch noch kaum voneinander unterscheidbaren "Pflänzchen und Thierchen ${ }^{70}{ }^{70}$ Wie dieser "Inbegriff des Wechselverkehrs" in größerem Maßstab funktioniert, demonstriert er am Beispiel des Waldes: Die »Organisation", d.h.: organische Bildung von "Zellstoff " geschieht "aus Kohlensäure und Wasser«, wobei diese um "Sauerstoff« "verarmt« werden, den der Wald »in die Luft zurück[gibt] «. ${ }^{71}$ In einem Rückblick zeichnet er sodann die Geschichte der Stoffwechselforschung nach: Die Entdeckungen von Joseph Priestley (1771), dass die "von den Pflanzen ausgehauchte Luft die Verbrennung unterhält", und von Jean Senebier (1783), »daß Sonnenlicht den Blättern die Fähigkeit verleiht, die feste Luft, die Kohlensäure, zu binden und daraus Lebensluft, den Sauerstoff, frei zu machen«, gehören demnach zu den wichtigsten Ergebnissen der wissenschaftlichen "Arbeit«, »die stetig dem Ziele zuschritt«. ${ }^{72}$

Dass das Sinngedicht mit dem Öffnen des Fensters und dem Hereinlassen von "Morgenglanz" und "Sommermorgenluft" anhebt, hat gewiss auch topische Gründe. Auch dass Reinharts Haare mit dem immerhin merkwürdigen Epitheton sschattig <elegt werden, mag mit der von Moleschott vorgebrachten Beobachtung, dass »dunkle[ ] Wohnungen« »bleichsüchtig« machen, zusammen gelesen werden, kann aber auch noch als Allgemeinerfahrung verbucht werden. ${ }^{73}$ Doch der Erzähltext hält auch dort mit, wo

70 Ebd., S. 9f.

71 Ebd., S. 13.

72 Ebd., S. 14-16.

73 Ebd., S. 22. 
für Moleschott »die Zahl allein die Probe für den richtig erkannten Zusammenhang«, nämlich das Verhältnis von Sonnenlicht und Produktion bzw. Assimilation von Sauerstoff und Kohlensäure liefert. Was die Lichtqualität angeht, so heißt es:

Die Strahlen, in welche das Sonnenlicht durch ein Prisma zerlegt wird, haben ungleiche Brechbarkeit und ungleiche Wirkung auf andere Naturkörper. [...] An den Grenzen des sichtbaren Farbenfeldes [...] stehen die rothen Strahlen als diejenigen, welche die geringste, und die violetten, welche die größte Brechbarkeit besitzen. An das violette Licht reihen sich noch stärker von ihrer Richtung abweichende Strahlen, für welche dem Auge die Empfänglichkeit abgeht. Sie verrathen ihre Gegenwart nur durch die chemischen Wirkungen, welche sie einzuleiten vermögen. [...] Draper [...] hat durch sorgfältige Versuche erwiesen, daß die Zersetzung der Kohlensäure durch die Pflanzen nur unter dem Einfluß der leuchtenden Strahlen vollzogen wird. Weder die chemischen, noch die erwärmenden Strahlen des Farbenfeldes fand er im Stande, die Ernährung der Pflanzen im Mindesten zu fördern. ${ }^{74}$

Im Sinngedicht heißt es modalisierend, dass Reinhart "womöglich das innerste Geheimnis" solcher Kristallkörper zu beleuchten versuche, was offenlässt, ob Reinharts »sinnreicher Apparat « und die Zahlenkolonnen nicht just der Bestimmung der Grenzen der verschiedenen Lichtfarben zur weiteren Erprobung ihrer biochemischen Wirkungen dienen. ${ }^{75}$

Solche Befunde aus seiner eigenen Produktion präsentiert jedenfalls Moleschott, auch wenn nicht die Qualität des Lichts, sondern nur die graduelle Quantität das Hauptkriterium bildet. Ihr Zweck ist die Erforschung des Einflusses des Sonnenlichts auf den »thierischen Stoffwechsel«: ${ }^{76}$

Läßt man Wasserfrösche bei gleichen Wärmegraden und auch unter denselben Bedingungen abwechselnd im Licht und in der Finsterniß athmen, dann findet man, daß sie in gleicher Zeit für gleiches Körpergewicht in diesem Falle viel weniger Kohlensäure liefern als in jenem. [...] So deutlich dieser Unterschied auch sprach, den ich als Ergebniß langer Versuchsreihen, theils an denselben, theils an verschiedenen, aber in allen Beziehungen möglichst ähnlichen Einzelwesen gefunden habe, ich durfte doch die Untersuchung nicht versäumen, ob die Erscheinung, welche sich als die Wirkung einer einzigen willkürlich veränderten Bedingung herausstellte, auch mit dem Wachsthum regelmäßig Schritt halten würde. Deshalb stellte ich mehr als neunzig Versuche an, bei welchen die Lichtstärke dadurch gemessen ward, da die Schwärzung mit ammoniakalischem Chlorsilber getränkter Papierstreifen, wie sie während der einzelnen Versuche in einer gegebenen Zeit das Licht erzeugte, mit einer von Künstlerhand sorgfältig angefertigten Farbenleiter verglichen wurde.

74 Ebd., S. 17f.

75 Keller: Das Sinngedicht (wie Anm. 22), S. 10.

76 Moleschott: Licht und Leben (wie Anm. 62), S. 24. 
Als ich dann alle Beobachtungen in zwei Hälften theilte, von welchen die eine auf die niederen, die andere auf die höheren Lichtgrade sich bezog, ergab sich, daß bei heller Beleuchtung beinahe ein Fünftel mehr Kohlensäure geliefert wurde, als im schwachen Licht. ${ }^{77}$

Nun weiß man Genaueres um das Schicksal des rätselhaften Frosches, der in Reinharts Laboratorium »in einem Glase [hockte] und [...] seines Stündleins [harrte] ${ }^{78}$ Neben der Bekräftigung des Zusammenhangs von Stoffwechsel und Lichtexperiment durch die näheren Anwendungs- und Anschlussmöglichkeiten sowie dem Streben nach zahlengestützter Empirie ließen sich auch noch etliche weitere Details zuweisen.

Eine erkenntnispoetische Pointe der narrativen 'Nachstellung` von Moleschotts Experimentalanlage von Licht und Leben besteht in der Verortung des Experimentators im Sinngedicht. Moleschott sieht Alltagserfahrungen wie jene, dass "Mastvieh" oder »Gänse«, die im Dunkeln gehalten werden, mehr Fett ansetzen, bestätigt, weil sie eben "weniger Kohlensäure ausathmen«. Dies gereiche "[h]öheren Lebensverrichtungen" jedoch "nicht zum Vortheil«, denn der »verzögerte Stoffwechsel wird gar bald von einer Abstumpfung der Nerven begleitet". Auch das hat Moleschott durch die Messung der "Reizbarkeit" bzw. des "galvanischen Nervenstroms und des elektrischen Gegentheils" geprüft und in "fünfhundert Vergleichsversuch[n] an Fröschen« »einen Vortheil auf Seiten des Lichts« gefunden, »der für die Nerven auf ein Sechstel, für die Muskeln auf ein Zehntel des durchschnittlichen Ausschlags der Magnetnadel geschätzt werden darf «. ${ }^{79}$

Die Augenschmerzen werden durch Reinharts Platzierung in einem Laboratorium ohne Licht und Frischluft analog zum Frosch im Glas und durch die Attribuierung mit sschattigen Haaren in einen kausalen Zusammenhang mit dem fehlenden Licht gebracht, auch wenn der Erzähler dann die Gründe "namentlich« im ständigen Wechsel zwischen Licht und Dunkel sieht. Der Text unterwirft Reinhart damit scheinbar konsequent Moleschotts materialistischer Bestimmung des Menschen als "Naturerzeugniß, das in der Erde wurzelt, wie die Pflanze, und wie die Pflanze von der Sonne durch Licht und Wärme Nahrung erhält und Wachsthum, Entwicklung und Rückbildung

77 Ebd., S. 23f.

78 Keller: Das Sinngedicht (wie Anm. 22), S. 10. Kaiser (Experimentieren oder Erzählen? [wie Anm. 17], S. 284) merkt dazu an, dass es sich um "eine verharmlosende Umschreibung seiner Bestimmung, für die damals üblichen galvanischen Experimente sein Leben zu lassen", handle, was tatsächlich auch Teil dieses Experiments sein kann, wie man gleich sehen wird, aber nicht einfach bloß topischen Charakter hat.

79 Moleschott: Licht und Leben (wie Anm. 62), S. $25 f$. 
ohne Wanken«. Dazu gehört auch die »Erfahrung, daß Keiner ungestraft die Grenze seiner Kraft überschreitet«. Entsprechend schränkt Moleschott die Freiheit des Willens ein auf die "Einsicht" in die "unwandelbare Nothwendigkeit«, die sich, »langsam zwar, aber Stück für Stück berechnen« lassen wird. Denn das »Streben, die Erkenntnis, welche unser Gefühl mit dem Naturgesetz in Einklang bringt, zu stärken, gehört unzertrennlich mit zur Willenskraft«. ${ }^{80}$

Im argumentativen Zirkel fallen Wille und Naturgesetz nur deshalb nicht in eins, weil das Ziel noch nicht erreicht ist, durch den Fortschritt aber unfehlbar erreicht werden wird. Dann würde es tatsächlich nur mehr die Tat geben, die Moleschott mit Faust an den Anfang setzt. Es ist dieser totale Determinismus, der auch die Zeit und den Zufall ausschaltet, gegen den Reinhart die "Kraft" setzt, die mit der Wortgeste "oder so was" dem Nichtwissen, der Unbestimmtheit Raum gibt. Auch wenn es sich bei der »Kraft«-Geste um eine 'Fehlleistung` Reinharts, des Erzählers oder gar des aus der Erinnerung referierenden Autors handeln sollte, so kann die oben aufgeschobene Deutung nun lauten, falsifiziert sie den im Experiment implizierten Determinismus. Erst recht lässt die folgende, von Willensakten, Unwillkürlichkeiten und Zufällen getaktete Handlung bis zum Aufbruch - die "unvermerkt" aufkommenden Vorstellungen von (weiblicher) Gestalt und Sprache, der Gang auf den Dachboden, der Griff nach Lessing mit seinem "unbedingte[n] gute[n] Wille[n] ohne Falsch", der Fund des Sinnspruchs und seine Interpretation als Kussexperiment ${ }^{81}$ - jegliche Frage nach der Freiheit oder Bedingtheit des Willens als sinnlos erscheinen. Die "Kraft» ist die begriffliche Grenzmarke, welche die Naturgesetzlichkeit unterbricht und als Metonymie des Unbestimmten einen Zeit-Raum des Lebens und Erzählens eröffnet.

\section{Zeit und Zufall}

Es ist oben anhand der verschiedenen Anachronismen bereits dargelegt worden, wie die Rahmenerzählung die lineare und kontinuierliche Zeit von Beginn an moduliert: Die Projektion der Darwin'schen Evolutionstheorie und die Verdichtung der Wissenschaftsgeschichte schaffen einen Raum

$\begin{array}{ll}{ }^{80} & \text { Ebd., S. 29f. } \\ & \text { Keller: Das Sinngedicht (wie Anm. 22), S. 12f. }\end{array}$ 
der Gleichzeitigkeit des Ungleichzeitigen, den das physische Symptom des Schmerzes in eine Eigenzeit des Lebens und Erzählens überführt. ${ }^{82}$ Durch die Zufälligkeit, die der Text hier spielen lässt, erscheint die Rahmenerzählung aus narratologischer Perspektive willkürlich. Dass das so prominent herbeizitierte "Gesetz der natürlichen Zuchtwahl ${ }^{83}$ schon hier über die offensichtliche Partnerwahl hinaus in Bezug auf tragende Theoriemomente mit inszeniert und reflektiert wird, ist bislang nicht beachtet worden.

Die snarratogene Modellhaftigkeit der Evolutionstheorie liegt u.a. in der Verkopplung von Organologie und Zeitlichkeit durch den Zufall: Sie ist eine Organologie, in der die Entelechie des Organismus durch die Kontingenz und durch überindividuelle biologische und physikalische Eigenzeiten dynamisiert wird. Die gesamte psychophysische Intentionalität und Kontingenz des Individuums, also der innere Wille und die äußeren Zufälle, treten mit einer sich ständig neu ausrichtenden Intentionalität und Kontingenz der biosozialen Gemeinschaft und der biologischen Art in Interaktion und diese wiederum mit den anderen biologischen Arten und physikalischen Vorgängen, so dass nur über einen bestimmten Zeitraum das Verhältnis von Beständigkeit und Wechsel als so etwas wie Intentionalität oder Determination betrachtet werden kann. Modus der Interaktion ist die Konkurrenz um die biologischen und vitalen Ressourcen, d.h. um deren Beschaffung, Verwertung, Vermehrung, Bewahrung und Weitergabe.

Darwin grenzt die natürliche Zuchtwahl von der künstlichen Zuchtwahl des Menschen ab, sei es in Bezug auf die Züchtung von Pflanzen und Tieren, sei es in Bezug auf das bewusste oder unbewusste Verhalten von Individuen und Kollektiven, wobei das Hauptkriterium die Dimension der Zeit bildet: Auch wenn der Mensch bei »Zuchttieren und Zuchtpflanzen durch Häufung individueller Unterschiede in bestimmter Richtung große Resultate hervorbringen« könne, so vermöge dies die "natürliche Zuchtwahl umso eher, als ihr unvergleichlich mehr Zeit zur Verfügung« stehe. ${ }^{84}$ Die modifizierende Übertragung einzelner oder mehrerer Elemente der Theorie auf die kurzfristigen sozialen, ökonomischen und politischen Verhältnisse der Gegenwart und die Ableitung von Handlungskonzepten mit einer beträcht-

82 Vgl. Michael Gamper/Helmut Hühn: Einleitung, in: Dies (Hg.): Zeit der Darstellung. Ästhetische Eigenzeiten in Kunst, Literatur und Wissenschaft, Hannover 2014, S. 7-23, hier S. 7-18.

83 Keller: Das Sinngedicht (wie Anm. 22), S. 9.

84 Charles Darwin: Die Entstehung der Arten durch die natürliche Zuchtwahl [1859], aus dem Englischen von Carl W. Neumann, Nachwort von Gerhard Heberer, Stuttgart 1998, S. 124, vgl. S. 100. 
lichen biopolitischen Reichweite sind durch diese Differenz keineswegs verhindert worden. Und gerade die referierten materialistischen Thesen zur Einheit von Kraft und Stoff beförderten solche Kurzschlüsse.

In der älteren Forschung ist man sich einig gewesen, dass Keller antidarwinistisch eingestellt war, wofür insbesondere Rothenberg die vielen direkten und indirekten Persiflagen der Zuchtwahl in den Sinngedicht-Novellen anführt. ${ }^{85}$ Die neueren Forschungen haben, wie mit Ajouri referiert worden ist, anhand des Grünen Heinrich gezeigt, wie Keller die Darwin'schen Erkenntnisse zu Zeit und Zufall in der Entteleologisierung des Erzählens umsetzt. Zuvor hat bereits Neumann in der Analyse des Pygmalionismus auf die positive Implementierung von Darwin'schen Theoremen im Sinngedicht aufmerksam gemacht: Die Kontingenz durchbricht gerade den Determinismus der Fortschrittsideologie, den die einschlägige Rezeption verabsolutiert, und dynamisiert die Zeit. Die eigentlich unzulässige Übertragung auf die menschlichen Verhältnisse, welche die Rahmenerzählung vornimmt, rechtfertigt sich durch den Gewinn an narrativer Komplexität und vitaler Dynamik. Was die ersten Seiten des Rahmenkapitels in nuce performieren, erhebt der Schluss zur expliziten Geste: In einer von Symbolik triefenden Szene beobachtet das Liebespaar auf seinem Gang durch die Natur, wie ein "starker Krebs" eine "große schöne Schlange" angreift, "vermutlich um sie anzufressen«. Mit einer partnerschaftlichen Aktion, in der Reinhart sein Naturwissen anwendet und Lucie ihre Kreaturphobie überwindet, befreien sie die Schlange vom Krebs, und Reinhart kommentiert: »Ja [...], es erfreut uns, in dem allgemeinen Vertilgungskriege das einzelne für den Augenblick zu schützen, soweit unsere Macht und Laune reicht, während wir gierig mitessen. ${ }^{86}$ Indem sie den Lauf der Natur mit ihrem »kleine[n] Rettungsabenteuer" willkürlich unterbrechen, schaffen sie jene Raum-Zeit, in der Leben und Erzählen einander hervorbringen, bevor die Natur sie einholt.

${ }^{85}$ Vgl. Rothenberg: Geheimnisvoll schöne Welt (wie Anm. 17).

86 Keller: Das Sinngedicht (wie Anm. 22), S. 325; vgl. Kaiser: Experimentieren oder Erzählen? (wie Anm. 17), S. 293. 